\title{
Measuring and interpreting transposable element expression
}

Sophie Lanciano and Gael Cristofari ${ }^{\dagger}$

University Côte d'Azur, Inserm, CNRS, IRCAN, Nice, France.

†e-mail: gael.cristofari@univ-cotedazur.fr

\begin{abstract}
Transposable elements (TEs) are insertional mutagens that contribute greatly to the plasticity of eukaryotic genomes, influencing the evolution and adaptation of species as well as physiology or disease in individuals. Measuring TE expression helps to understand not only when and where TE mobilization can occur, but also how this process alters gene expression, chromatin accessibility or cellular signalling pathways. Although genome-wide gene expression assays such as RNA-sequencing include transposon-derived transcripts, the majority of computational analytical tools discard or misinterpret TE-derived reads. Emerging approaches are improving the identification of expressed TE loci and helping to discriminate TE transcripts that permit TE mobilization from gene-TE chimeric transcripts or pervasive transcription. Here, we review the main challenges associated with the detection of TE expression, including mappability, insertional and internal sequence polymorphisms, and the diversity of the TE transcriptional landscape, as well as the different experimental and computational strategies to solve them.
\end{abstract}




\section{Introduction}

Transposable elements (TEs) are mobile genetic elements that form a large fraction of eukaryotic chromosomes, ranging from $12 \%$ of the Caenorhabditis elegans genome to up to $85 \%$ of the maize genome ${ }^{1}$. Consistently, genome size largely reflects TE copy number ${ }^{1}$. TEs are insertional mutagens and major drivers of genome evolution, acting both in the germline and in select somatic tissues. Their influence on host adaptation and disease, such as tumorigenesis or neurodegenerative diseases, has been extensively documented ${ }^{2-6}$.

TEs belong to different classes, depending on their molecular mechanism of replication, with a major distinction being made between retrotransposons and DNA transposons (Fig. 1). Transcription is the first step in the replication of retrotransposons. Retrotransposon RNA can serve as a template for both the translation of retrotransposon proteins and for reverse transcription, a process leading to the formation of a new DNA copy that is inserted into the host genome. While transcription is also important for DNA transposons - it enables transposase expression, the protein required at the excision and reintegration steps - we focus here mainly on retroelements because of their specificities. Thus, although many posttranscriptional mechanisms can suppress TE mobilization, transcription is a prerequisite for their proliferation and mutagenic activity.

Many TEs are molecular fossils, remains of past mobilization waves that occurred millions of years $\mathrm{ago}^{7}$. These ancient TEs have accumulated inactivating mutations and truncations that prevent their mobilization in modern genomes (Fig. 2a), but can still be transcriptionally active, with potential consequences for the host genome ${ }^{8-10}$. Independently of its role in transposition, TE expression itself - through the transcript, the act of transcription itself, or subsequent TE replication intermediates - can regulate gene expression ${ }^{11-13}$ and chromatin accessibility ${ }^{14}$, activate cellular signalling pathways, such as the interferon response $^{15}$ or RNA interference (RNAi) responses ${ }^{16}$, and trigger ageing ${ }^{17}$ or antiviral activities $^{18}$.

With a few exceptions, transcription along the length of the TE unit is usually driven by an internal promoter, which is mobilized concomitantly with the element itself. This ensures that the newly inserted TE possesses its own promoter and does not depend on the presence of a cellular promoter near its insertion site. However, because TEs can contain multiple cis-acting sequences (for example, sense and antisense promoters, acceptor and donor splice sites, or polyadenylation signals), be fragmented, and insert into or in the vicinity of genes, their transcriptional activity is often intertwined with that of genes. Hence, autonomous TE unit transcription [G] can easily be confounded with TE-chimeric transcripts [G] or with the expression of the gene into which a given TE is inserted, a situation referred to as cotranscription [G], which is also known as read-through transcription. 
In summary, TEs are repeated and interspersed, polymorphic [G], and may overlap with genes, creating complex transcripts initiated from TE or gene promoters. These unique features hinder the analysis of TE expression with standard methods developed to monitor gene expression, whether based on hybridization or sequencing, such as reverse transcriptionquantitative polymerase chain reactions (RT-qPCR), northern blotting, microarrays or RNA sequencing. Careless application of these methods can lead to overestimation or underestimation of TE expression; erroneous conclusions regarding TE reactivation; misinterpretation of their impact on the host transcriptome; or simply overlook their involvement in the process under study. However, dedicated algorithms, multi-omics approaches and advances in sequencing technologies have recently improved the quantification and interpretation of TE expression, providing new insights into host-TE interactions.

Here, we first outline the three major TE characteristics that hamper the study of TE expression. We then present the main experimental approaches used for the quantification and interpretation of TE expression before we highlight how recent advances can overcome the main challenges posed by the particularities of TEs, as well as existing limitations. Finally, we discuss the perspectives raised by new algorithms and long-read sequencing technologies.

\section{Challenging features of TEs}

\section{TE sequences are repetitive and interspersed}

At the time of integration into the genome, a new TE copy is identical to its source - or progenitor - copy. Nevertheless, in the absence of positive selection [G] , the internal sequence of TEs diverges progressively after integration through random mutations and other forms of alterations ${ }^{19}$ (Fig. 2). For simplicity, families that are currently, or were recently, active are often referred to as 'young families', whereas those with a higher degree of divergence towards consensus are referred to as 'old families' (Fig. 2). However, young and old TE families are relative concepts that depend on the investigated process. For instance, in humans, 'young' long interspersed element 1 (LINE-1 or L1) families may refer to the human-specific L1HS family when studying disease-causing insertions, but may include older families such as L1PA2 to L1PA5, which expanded approximately between 3-20 million years ago, when investigating primate evolution ${ }^{20}$.

The number of active families within a given species, as well as the number of active progenitor elements within these families, is highly variable ${ }^{21}$. In humans, only the youngest TE subtypes, Alu, L1 and SVA elements (retrotransposons composed of short interspersed elements (SINEs), variable number tandem repeats and Alu sequences), can still actively retrotranspose ${ }^{22}$. However, Alu and SVA are non-coding sequences and depend on L1 activity, as only the latter encodes the retrotransposition machinery. In fact, it is estimated that only 
80-100 L1 elements among the hundreds of thousands copies present in the human genome are retrotransposition-competent (whether expressed or not), all of which belong to the family of human-specific L1 (L1HS) ${ }^{23}$. Of these, only 20 are likely responsible for most ongoing L1 activity $^{23-27}$. Indeed, mammalian retrotransposons have been amplified by successive waves of retrotransposition of a small number of master copies that eventually become inactivated by mutations or silenced by epigenetic mechanisms ${ }^{20,28-31}$. As a result, internal diagnostic SNPs can be found that differentiate TE families or even lineages within a given family ${ }^{26,32,33}$.

In other vertebrates, insects and many plant species, many more TE families seem concurrently active compared with humans ${ }^{2,21,34,35}$. For example, in Drosophila melanogaster, the majority of TE families including DNA transposons (for example, Pogo and $P$ element), LTR-retrotransposons [G] (for example, Copia and Gypsy) and non-LTR retrotransposons (that is, LINE-like elements such as TART and Jockey) are likely to possess active members, and approximately $30 \%$ of all individual copies are considered capable of transposition ${ }^{36}$. However, it seems that in some of these organisms, such as Drosophila species, individual TE families have often not expanded to the copy numbers reached in mammals, possibly as a result of short generation time and rapid genomic turnover ${ }^{37-39}$. Internal fertilization and body temperature may also be important factors to explain such differences (discussed $\mathrm{in}^{21}$ ).

To summarize, older TEs have accumulated mutations, diverged over time and tend to become unique, whereas younger TEs are almost identical to each other (Fig. 2). The presence of old and recent families of evolutionary-related retrotransposons in the same genome increases the difficulty of distinguishing active from inactive families.

\section{TEs are polymorphic elements}

The ongoing activity of some TE families leads to insertional polymorphisms, that is, the presence or absence of a TE at a given locus within a species or within restricted populations. Some of these polymorphisms can even be private to a single individual. Various specialized wet-lab approaches and bioinformatics tools have been developed to identify mobile element insertions (reviewed $\mathrm{in}^{40-42}$ ). In humans, for example, $20 \%$ of all inherited structural variants result from new TE insertions ${ }^{43}$. Considering only L1s, two human individual genomes differ, on average, at 285 sites with respect to $L 1$ insertion presence or absence ${ }^{44}$. In mouse, the combined activity of $L 1$ and endogenous retroviruses (ERVs), such as the intracisternal $A$ particle (IAP) and early transposon (ETn)/Mus musculus type D (MusD) families, leads to even more TE insertional polymorphisms than in humans ${ }^{45-48}$.

At the scale of animal or plant natural populations, the extent of this type of variation seems to be considerable ${ }^{35,43,49-51}$. For example, in natural populations of the flowering plant Arabidopsis thaliana, TEs are strongly active, and thousands of TE insertional polymorphisms involving a hundred of different TE families have been identified ${ }^{35}$. Polymorphic TEs with low 
allele frequency tend to be among the most active elements since they inserted recently relative to the population history and, therefore, have little or no alteration ${ }^{24}$. Their mobilization can be influenced by environmental and genetic factors, and some of them show signatures of positive selection ${ }^{51,52}$, whereas others have detrimental effects and are implicated in disease $e^{5,33,53,54}$. Thus, it is critical not to dismiss polymorphic TEs when analysing TE expression. Finally, in addition to these insertional polymorphisms, the internal sequence of a given TE locus may also contain SNPs that differ from one individual to another and can alter their retrotransposition potential ${ }^{31,55}$.

\section{TE transcripts are diverse}

TE transcripts used as template for reverse transcription. Retrotransposon transcription is the starting point of the retrotransposition process. The synthesized RNA species that serve as canonical templates for productive reverse transcription are called TE unit-length transcripts [G] (also referred to as full-length, proper or genomic transcripts). Transcription is initiated from an internal Pol II promoter contained in the LTRs for LTR-retrotransposons and ERVs, or in the 5' UTR for LINEs ${ }^{56,57}$ (Fig. 3a). SINEs can have either internal Pol III promoters (for example, Alu and MIR) ${ }^{58}$ or Pol II promoters (SVA elements) ${ }^{59}$. Transcription can end upon recognition of a polyadenylation signal located in the 3' LTR (in the U3 or R segment) for LTRcontaining retroelements or in the $3^{\prime}$ UTR for LINEs ${ }^{60,61}$. Alternatively, termination can occur in the downstream flanking sequence. For example, Alu elements do not contain a Pol III termination signal, which consists of a simple $(\mathrm{T})_{4}$ tract, but transcription will stop as soon as this motif is reached in the flanking sequence ${ }^{62}$. Similarly, L1 elements have a weak polyadenylation signal, leading to a significant fraction of 3' readthrough ${ }^{63,64}$. The fraction of these 3'-extended L1 RNAs varies and might depend on the poly $(\mathrm{dA})$ length of the element ${ }^{65}$. These extended RNA species can be used as a template for reverse transcription as efficiently as unit-length transcripts, leading to the retrotransposition of sequences derived from L1 3' flank to new genomic locations ( $3^{\prime}$ transduction) ${ }^{64,66,67}$. Similarly, $L 1^{68,69}$ or SVA ${ }^{59,70}$ can be transcribed from a promoter present in their 5' flank, leading to 5 ' transductions when reverse transcribed. Note that 3'-readthrough refers to transcripts initiated from the L1 promoter but extending beyond its polyadenylation signal and ending in the 3 '-flanking sequence. This process is distinct from readthrough transcription, which corresponds to passive cotranscription of TE sequences included in genes, initiated from genic promoters. However, the $\mathrm{R} 2$ group of non-LTR retrotransposons is a notable exception to this scenario. These elements specifically integrate into ribosomal DNA (rDNA) and are co-transcribed with rDNA units. The R2 RNA is then cleaved from the co-transcript by a self-cleaving ribozyme positioned it its 5'end $^{71}$. 
Short TE transcript isoforms. In addition to full-length retrotransposon RNA, shorter TE transcript isoforms can be synthesized upon premature polyadenylation or splicing ${ }^{57,72-74}$, and can result from cellular regulatory mechanisms, such as Piwi-interacting RNA (piRNA)-guided alternative splicing ${ }^{75}$. Short TE transcript isoforms may encode proteins with significant biological activities. For example, human L1 can undergo splicing into a subgenomic RNA containing only ORF2p, a protein with endonuclease and reverse transcriptase activities ${ }^{74}$. On its own, this protein cannot support L1 retrotransposition, which also requires the expression of ORF1p from the full-length transcript, but it can mobilize Alu or SVA elements in trans and can trigger DNA damage ${ }^{76}$. Similarly, internal transcripts of the Ty1 retrotransposon in Sacchomyces cerevisiae encode dominant-negative forms of Gag, the main constituent of the virus-like particles, which limit its retrotransposition ${ }^{77}$. Retrotransposons also frequently contain antisense promoters, although they are probably not a major determinant of retrotransposon unit transcription ${ }^{78-84}$.

TE internal promoter integrity. The autonomous transcriptional capacity of retrotransposons depends on the presence and integrity of their promoter. However, LINE retrotransposons are frequently 5'-truncated at the time of insertion due to the resolution of the integration process and likely intervention by the DNA repair machinery ${ }^{85,86}$ (Fig. 3a). L1 promoter activity can also be lost by splicing of the L1 RNA within the 5 '-UTR before integration ${ }^{87}$. For example, of the 500,000 L1s present in the human genome, only 5,000 are full-length and thus include the internal 5'-UTR promoter typical of these elements ${ }^{23,88}$. Conversely, LTR-retrotransposons often undergo ectopic homologous recombination between their two LTRs (Fig. 2a, 3a), resulting in the complete elimination of coding regions, but leaving an intact solo-LTR with all its original cis-regulatory sequences ${ }^{57,89-92}$.

Chimeric TE RNA species and pervasive transcription. The retrotransposon transcription landscape is made more complex by interactions between the transcription units of genes and those of TEs, leading to chimeric transcripts, in which a fragment or all of the TE is incorporated into the mature mRNA ${ }^{93}$ (Fig. 3). Solo-LTRs, as well as antisense L1 promoters, often drive the synthesis of long non-coding RNAs (IncRNAs) ${ }^{94-98}$. They can also act as alternative promoters for cellular genes, leading to chimeric TE transcripts (Fig. 3b), often in conjunction with splicing events ${ }^{57,78,82}$. Alternatively, TEs or TE fragments can be incorporated into spliced mRNA by co-transcription with a cellular gene into which they are inserted. This can occur when TEs are inserted in exons (often corresponding to the 3'-UTR), or when TEs are inserted in introns but a fragment of their sequence is exonized by splicing (Fig. 3b). This scenario is far from anecdotal, since more than a third of human protein-coding transcripts contain an exon of TE origin (mainly in their UTRs), as do three quarters of human IncRNAs ${ }^{94,95}$. As a 
consequence, an apparent change of TE expression levels may simply reflect variation of the expression of the gene into which a member of this particular TE family is inserted.

Given the abundance of TEs in eukaryotic genomes, especially in intergenic regions and introns, pervasive transcription [G] and pre-mRNAs can represent a very large fraction of all TE-containing RNA species, even though each locus contributes only minimally to the whole transcriptome ${ }^{99,100}$ (Fig. 3b). For example in humans, $>99 \%$ of L1-derived RNAs originate from co-transcription or pervasive transcription and do not reflect transcription from L1 unit-transcripts ${ }^{99}$. The biological impact of pervasive transcription is not well understood, but part of it is involved in the production of IncRNAs ${ }^{94,95,101,102}$ and enhancer-associated RNA $(\mathrm{eRNA})^{103}$.

Double-stranded TE RNA. The considerable diversity of TE-containing transcripts can lead to the formation of double-stranded RNAs (dsRNA) through complementarity between sense and antisense transcripts (Fig. 3). These can arise through convergent and overlapping transcription or through annealing of transcripts from different loci sharing homologous TE sequences. Synthesis of dsRNA species can trigger RNA interference and silencing of TEs in a wide variety of organisms ${ }^{11,83,104-109}$. TE-derived dsRNA transcripts can also be formed by annealing of a genic transcript with an antisense RNA initiated from intra- or intergenic TEs, inducing the repression of the gene ${ }^{110}$ or silencing of the implicated $\mathrm{TE}^{111}$. Distinct cellular transcripts containing TE in opposite orientation can also regulate each other by Staufenmediated RNA decay ${ }^{112}$. Similarly, DNA demethylating agents, such as those used in cancer chemotherapy, induce the expression of TE-derived dsRNAs that activate antiviral defences and interferon response pathways ${ }^{113,114}$.

To summarize, the transcriptional landscape of TEs is not limited to unit-length TE transcripts that will serve for retrotransposition but includes a number of chimeric or pervasive transcripts, originating from TE promoter activity or from passive co-transcription. Overall, these RNA species can significantly influence cell physiology independently of TE mobility.

\section{Measuring TE expression}

Many molecular and computational tools are now available to assess TE expression, but the strategy must be guided by well-defined underlying biological questions and hypotheses. Aspects of TE biology that are often investigated include: whether TEs competent for mobilization are expressed, which may lead to new insertions; whether TEs have a functional impact on genes; and whether biologically active molecules derived from TEs are synthesized (that is, dsRNAs, small RNAs or TE proteins). In the following section, we list conventional and genome-wide approaches available to measure and understand the expression of TEs and explain how they can help study specific facets of their biology. 


\section{Conventional approaches}

Although the use of sequencing techniques is growing exponentially, conventional molecular biology approaches are still commonly used to study TEs, some providing unique information that is not available with genome-wide approaches.

Detection of TE-derived transcripts. RT-qPCR is commonly used for measuring the transcriptional level of TEs but presents several major limitations. First, because the starting material is generally total RNA, including pre-mRNA, autonomous and passive transcription are confounded (Box 1). Second, it is often difficult to design probes and primers truly specific to a given TE family. Third, the sequence of the amplified fragment is unknown and may come from defective copies with mutations or truncations, or from non-unit-length transcripts ${ }^{99}$. Instead, northern blotting may reveal the size distribution of TE-derived transcripts and the potential presence of full-length TE transcripts ${ }^{115,116}$, although cross-hybridization of probes between related families is possible. Finally, reporter gene knock-in can be used to measure the autonomous transcription of individual TE loci and can be parallelized. This approach has been used to test the transcriptional activity of each individual Ty1 retrotransposons present in a laboratory strain of $S$. cerevisiae ${ }^{117}$, but is difficult to generalize.

Detection of TE proteins. Internal TE mutations that prevent the translation of functional TE proteins $^{23,88}$ and post-transcriptional regulation by cellular factors limit retrotransposition downstream of TE transcription ${ }^{75,118-121}$. With respect to this issue, western blotting and immunofluorescence experiments are complementary approaches that can help to evaluate the expression of the mobilization machinery itself. However, the use of protein-based approaches is limited by the availability of specific, sensitive and well-validated reagents, the potential cross-reactivity of antibodies between related families of TE, and the frequent need for large quantities of starting material. Similarly, purification or direct visualization by electron microscopy of replicative complexes (for example, the ribonucleoprotein particle or virus-like particles) represent direct means of detecting assembled replication intermediates and, thus, a certain level of functionality ${ }^{122-125}$.

Altogether, some of these techniques are useful for testing the overall expression of selected families of TEs and may provide unique insights (for example, length and coding capacity of TE transcripts, potential of assembled complexes), but other strategies are needed to obtain an unbiased and genome-wide view of TE expression.

\section{Genome-wide analysis of TE expression}

Although past attempts have been made to take advantage of general-purpose or specialized microarrays, they have not been widely adopted to analyse TE transcription ${ }^{126-130}$, likely owing 
to difficulties in designing short and specific probes. They have now been largely supplanted by deep-sequencing technologies. However, constraints of short-read sequencing and the specific features of TEs detailed above mean that TE transcription cannot be analysed in the same way as gene transcription. Hence, the number of TE-dedicated computational approaches and tools is rapidly increasing (Table 1), and selecting one can be challenging. Most genome-wide approaches use RNA-seq data, but they mainly differ on: their mapping strategy (the use of uni- and multi-mapping reads) and their resolution (family or locus-specific level); their strategy to take into account TE polymorphisms; their ability to distinguish autonomous from co-transcription and pervasive transcription; their ability to discover and/or quantify chimeric transcripts; and the analysis of other TE-derived transcripts such as dsRNA and small RNAs.

\section{Tackling TE-specific challenges}

\section{Mappability}

A practical consequence of TEs being highly repeated sequences, as well as evolutionaryrelated TE families being present in the same genome, is that short sequencing reads originating from TEs can often map equally well at different positions in the genome (Fig. 4a). These reads are referred to as 'multi-mappers [G]' and, therefore, their locus of origin cannot be unambiguously defined. Similarly, primers or probes can cross-hybridize to multiple copies or related families. A simple strategy to circumvent the mappability (Box 2) problem when studying the TE transcriptome is to map reads against the reference genome and keep only the unique reads, then aggregate the counts for each family. Keeping only uniquely mapping reads, known as 'uni-mappers [G]', can provide satisfactory estimates for the expression of old TE families ${ }^{99,131}$. Nevertheless, this approach should be avoided as it tends to greatly underestimate or even eliminate the signal associated with young TE families, that is, those which are still mobilization-competent (Fig. 4). Consequently, the signal reflects more closely the mappability (Box 2) of the element rather than its transcript level ${ }^{132}$. This effect can be somewhat mitigated by increasing read length and using paired-end libraries. Only $68 \%$ of annotated human TEs are uniquely mappable with short reads of $50 \mathrm{bp}$, but $88 \%$ are mappable with 100 bp-long reads ${ }^{133}$. However, even with $2 \times 100$ bp paired-end libraries, less than half of the reads emanating from the youngest human $\mathrm{L} 1$ family, L1HS, or from the 25 youngest TE families in the mouse genome are uniquely mapped ${ }^{134}$. Thus, multi-mapping reads are a challenge for recently or currently active TE families, but less for older families, at least with commonly used short-read sequencing technologies and experimental conditions.

By contrast, mapping reads against a library of consensus sequences, such as Repbase $^{135}$, will directly provide aggregated TE counts by family and may be useful for the 
youngest elements. However, as mapping efficiency decreases for old elements that are more divergent from their consensus sequence, the stringency of alignment must be relaxed to tolerate more mismatches (Fig. 4). As a result, the mapping of non-TE reads or reads from related TEs can be forced to the provided sequences alone, leading to overestimates of the read count of this family. TEtools is a declination of this approach in which consensus sequences are replaced by the entire set of genomic repetitive sequences ${ }^{136}$. While this method resolves the mapping bias relative to TE age, it still tends to overestimate some TE counts by forcing non-derived fragments to map to TE sequences ${ }^{134}$. A missing aspect of these TE-centred reference approaches is the possibility to distinguish co-transcription from TE unit transcription, with the consequence of overestimating TE family transcription levels for both young and old elements. Other limitations are that inter-family ambiguities still occur, the number of loci expressed is unknown, and most reads remain unmapped, complicating normalization and sample-to-sample comparisons. Nevertheless, when studying species for which a reference genome or transcriptome is not available, they may be the only options for obtaining a first glimpse of the TE transcriptome ${ }^{137-139}$.

Mapping reads against a reference genome rather than consensus sequences provides a better picture of TE transcription. Many tools take advantage of general usage mapping softwares, such as Bowtie $2^{140}$, BWA ${ }^{141}$, TopHat ${ }^{142}$ or STAR ${ }^{143}$, and first discriminate uniquely mapped reads from multi-mapped reads. Then, the strategies differ on the fate of multi-mapped reads. For example, RepEnrich realigns multi-mappers on a pseudo-genome containing all annotated and concatenated repeats of the genome of interest, providing a fractional value inversely proportional to the number of families with a match for this read ${ }^{144}$. This approach seems to underestimate the expression levels of young elements in contrast to a strategy that randomly assigns multi-mappers to a genomic location among the best scoring loci $^{134}$ (Fig. 5a). This bias may also result from Bowtie 1, the underlying mapping software recommended by RepEnrich, which cannot align discordant reads or reads with small insertions and deletions (indels), and only outputs a limited number of fraction of all possible positions for multi-mapping reads ${ }^{145}$. By contrast, the TEcandidates pipeline first performs de novo transcriptome assembly to identify potentially expressed TE loci, then masks nonexpressed ones in the reference genome, and finally remaps multi-mapping reads on this masked genome with less mapping ambiguity ${ }^{146}$. However, the ability of this pipeline to properly assemble TE transcriptomes, or to identify expressed loci among young TEs, has not yet been evaluated.

Another set of strategies consists of statistically reassigning multi-mapped reads according to the quantification of uniquely mapped reads ${ }^{131}$ (Fig. 5a). The application of the expectation-maximization (EM) algorithm to this problem is a generalization of this rescue method, in which reassignment is achieved reiteratively with read count of both uni- and multi- 
mappers at each step being used to reassign multi-mappers at the following step, until convergence is achieved. Initially developed to identify isoform-specific transcription in RSEM $^{147}$, it was subsequently incorporated in TEtranscripts ${ }^{148}$ and multiple other software for TE transcriptome analysis (Table 1). Interestingly, EM-based algorithms have the potential to provide insights also into the structure and origin of TE transcripts (discussed below). Although TEtranscripts' quantification is limited to the family-level, more recent tools such as SQuIRE ${ }^{145}$ or Telescope ${ }^{149}$ can provide locus-specific estimates, albeit with reduced confidence regarding the youngest TE subfamilies ${ }^{145}$. Pseudo-alignment on a model transcriptome, as implemented in Kallisto ${ }^{150}$ or Salmon ${ }^{151}$, can be a faster alternative to genome alignment (Fig. 5b). In short, pseudo-alignments test the compatibility of read k-mers [G] with the k-mers extracted from all possible paths of a transcriptome de Bruijn Graph. Both SalmonTE ${ }^{152}$ and REdiscoverTE ${ }^{153}$ apply this method for the quantification of TE transcription. However, the SalmonTE transcriptome model is based on Repbase consensus sequences, whereas REdiscoverTE uses annotated TE sequences extracted from a reference genome and introduces alternative transcript models for co-transcription (see below). Thus, REdiscoverTE may provide more accurate quantification of full-length unit transcripts when TE genome annotations are available.

To summarize, random read assignment on best hits or EM-based softwares can provide consistent TE expression analysis at the family level. Nevertheless, identifying the exact expressed loci remains approximate, particularly for the youngest TE families.

\section{TE sequence and insertional polymorphisms}

In practice, the analysis of RNA-seq data invariably begins by mapping reads to a reference genome or transcriptome, which contains neither insertional polymorphisms nor internal sequence polymorphisms. For the youngest TE families, even uni-mapping reads can be ambiguous, as they may originate from an expressed locus not represented in the reference genome $^{33,154}$ (Fig. 4b). Instead, they would map to the source element (if it is itself included in the reference genome) or to a closely related element. Furthermore, discrimination of closely related sequences relies on a few internal and diagnostic SNPs in each TE locus. Sequence polymorphisms between individuals, as well as sequencing errors, add additional levels of variation, increasing mapping ambiguity of uni-mapping reads (Fig. 4c, discussed in ${ }^{132}$ ).

Although none of the methods described above take into consideration these various forms of polymorphisms, several elaborate solutions have been tried for human L1 elements. Philippe et al. first mapped the location of all full-length L1HS in the sample of interest by targeted DNA sequencing (ATLAS-seq), then identified among them the expressed copies by a signature combining active histone marks - that is, the histone 3 lysine 4 trimethylation (H3K4me3) chromatin immunoprecipitation followed by sequencing (ChIP-seq) signal — just 
upstream of the element and 3' readthrough transcription just downstream of $\mathrm{it}^{154}$. In this approach, multi-mapping reads internal to the TE sequence are completely ignored. It is currently unclear if all L1HS loci have the potential to generate 3'-readthrough, which may represent a limitation of this approach. Indeed, some L1HS loci can be identified by $\mathrm{L}_{1 \mathrm{EM}}{ }^{65}$, a software focused on L1 and based on the EM algorithm, as expressed but without readthrough in the flanking sequence. Nevertheless, it is also possible that these reads actually originated from related non-reference insertions not represented in the L1EM index and were thus misplaced. As more and more catalogues of polymorphic TE become available $26,43,155,156$, the initial mapping step may become avoidable in the future ${ }^{99}$.

An alternative strategy was developed to identify and measure the expression of a polymorphic L1HS element responsible for a driver mutation in colon cancer ${ }^{33}$. This approach also starts by mapping all L1HS elements in the genome of the patient by whole-genome shortread sequencing. Next, the entire set of non-reference full-length elements was fully sequenced by combining long-range PCR, and Sanger or long-read (PacBio) sequencing, enabling the inference of a unique signature of diagnostic SNPs for each of these $6 \mathrm{~kb}$ loci. Finally, RNA-seq reads spanning these internal polymorphisms were used to estimate the relative expression of each locus.

Of note, the coverage of SNPs diagnostic for a TE family rather than for a locus can also be used to estimate relative family-level expression ${ }^{154}$. Altogether, obtaining locus-specific expression of non-reference TE copies remains a difficult and work-intensive objective that can to date be achieved only by multi-omics approaches (Table 1).

\section{Co-transcription and pervasive transcription}

When studying retrotransposition or its transcriptional regulation, distinguishing autonomous TE unit-length transcription from passive co-transcription with genes, including intron retention, or from pervasive intergenic transcription, is not a trivial task. Indeed, the vast majority of TEderived RNA-seq reads originate from co-transcription or pervasive transcription ${ }^{99,100}$. Recent efforts have tackled this problem. ERVmap uses an ad hoc curated database of full-length ERV elements and applies stringent criteria to filter ambiguous reads and low-mappability regions in ERVs ${ }^{157}$. Thus, this approach provides count quantification for each annotated fulllength ERV and partially integrates the coding capacity of the element but without differentiating autonomous from pervasive transcription. By contrast, TeXP' ${ }^{100}$ applies a correction based on mappability signatures from simulated pervasive and autonomous transcription to estimate family-level expression. Other corrective approaches include a modification of TEtranscripts that reduces the read count of intronic TEs proportionally to the coverage of their surrounding introns ${ }^{132}$, or to discard reads that overlap both TE and known coding or non-coding transcripts ${ }^{158}$. REdiscoverTE explicitly models autonomous and co- 
transcripts in the indexed transcriptome for Salmon pseudo-alignment ${ }^{153}$. Finally, L1EM includes models for autonomous sense and antisense transcriptions, passive co-transcription and 3' readthrough at each locus and can provide locus-specific expression values ${ }^{65}$ (Fig. 4c). However, although generalizable, its current implementation focuses only on L1 elements. Manually curated data sets, as published for human $\mathrm{L} 1 \mathrm{~s}^{99,159}$, will be useful to further compare and benchmark these recently developed software programs.

Identifying active promoters, either by genome-wide mapping of transcriptional start sites (TSS) using CAGE (Cap Analysis of Gene Expression) ${ }^{160,161}$ or RAMPAGE ${ }^{162}$, or by integrating chromatin modifications ${ }^{154}$, can also help to distinguish autonomous from passive TE transcription. Alternatively, 5' or 3' RACE (rapid amplification of cDNA ends) coupled to Sanger or high-throughput sequencing can define or confirm the boundaries of TE-containing RNA molecules and provide information on their locus of origin ${ }^{99,154,163-165}$. However, RACE experiments are not quantitative.

\section{TE-chimeric transcripts}

TEs nearby or within genes can provide alternative promoters or polyadenylation signals, as well as alternative splice acceptor and donor sites, which can profoundly alter gene expression patterns of the host ${ }^{80,160,166-173}$. TE-chimeric transcripts are defined by a portion of the mature transcript containing a TE fragment (Fig. 3c). Detecting these alternative transcripts, rarely included in common gene model datasets such as Refseq or GENCODE, relied initially on expressed sequence tag (EST) database computational screening against consensus repeat libraries $^{78,79,174-178}$. More recently, tools such as CLIFinder ${ }^{179}$ and LIONS ${ }^{180}$ combined split reads and discordant read pairs in RNA-seq paired-end libraries to systematically identify onco-exaptation events, where a TE provides an alternative promoter to a cellular gene leading to a novel oncogene or tumor suppressor gene isoform. TopHat-Fusion detects reads spanning gene and TE junctions to identify chimeric transcripts and can apply to both single and paired-end libraries ${ }^{181}$, but the number of false-positives is higher with single-end libraries. In addition, de novo transcriptome assembly can successfully identify chimeric TE-transcripts, such as those leading to the expression of oncogenes ${ }^{173}$ or cancer-specific antigens ${ }^{172}$, in a wide range of tumours. Techniques such as $\mathrm{CAGE}^{160,161}$ or RAMPAGE ${ }^{162}$ also permit detection of possible IncRNAs. Finally, different strategies were developed to associate expressed TEs with a modification of nearby gene expression. For example, NearTrans associates differentially expressed TEs with differentially expressed genes ${ }^{182}$, and TEffectR is an $R$ package based on a linear regression model intended to statistically associate TE transcription with the expression of nearby genes ${ }^{183}$. 


\section{TE-derived dsRNA and small RNA}

TE-derived dsRNA can lead to gene or TE silencing or to activation of the interferon response. Thus, quantifying pervasive transcription across genes or TEs is sometimes precisely what is being sought, and can be achieved by calculating the ratio between sense and antisense RNA at the features of interest in directional RNA-seq data ${ }^{111}$. More specific approaches have been developed such as dsRNA-seq ${ }^{184}$, which enriches dsRNA by digestion of single-stranded RNA and immunoprecipitation of dsRNA with a sequence-independent anti-dsRNA antibody, followed by sequencing. This approach was originally developed to identify viral dsRNAs. Candidate dsRNA-producing loci can be tested by RT-qPCR upon mild RNase A digestion, as dsRNA is more resistant than single-stranded RNA. This approach was used to confirm the presence of ERV dsRNAs induced upon treatment of cancer cells by demethylating agents ${ }^{113}$.

Similar to TE-derived dsRNAs, small RNAs (sRNAs), including miRNAs, shortinterfering RNAs (siRNAs) or piRNAs, play central roles in regulating TEs ${ }^{185}$. Some challenges are shared by both sRNA-seq and mRNA-seq analyses, such as mapping ambiguity or quantification ${ }^{186}$. However, sRNA-seq analysis in the context of repeated sequences has other specificities that are detailed elsewhere ${ }^{186,187}$.

\section{Future directions}

In the near future, we anticipate that recent experimental or computational advances may greatly facilitate the study of TE expression. Graph-based mapping ${ }^{188-190}$ has emerged as a new strategy to incorporate genetic variation (SNPs, indels and structural variants) found in the population into expanded model genomes, or pan-genomes, instead of consensus- or individual-based reference genomes ${ }^{191}$. Although not yet applied to TEs or to RNA-seq, this approach could reveal the expression of polymorphic TEs, as well as reduce mapping errors due to their absence in conventional reference genomes.

So far, mass spectrometry approaches to study TE expression have been only minimally exploited but recent results seem promising. For example, by using a strategy named proteomics informed by transcriptomics (PIT), which combines de novo RNA-seq assembly with proteomics data, a repertoire of active TE has been characterized in the poorly annotated mosquito (Aedes aegypti) genome ${ }^{192}$. This proteomic approach has high potential to identify biologically active proteins derived from TEs and to provide an overview of the transposition activity in a given condition or sample (reviewed $\mathrm{in}^{193}$ ). Similarly, mass spectrometry approaches and mining mass-spectrometry databases has permitted to validate the presence of predicted chimeric TE-derived peptides in tumours or primate embryonic stem cells ${ }^{153}$ or to confirm L1 expression in human cancers ${ }^{194}$. 
Single cell RNA-seq (scRNA-seq) experiments open the possibility to evaluate TE transcriptional heterogeneity in cell populations, especially in cancer tissues or in the brain, which could provide new insights into the mechanisms of TE activation ${ }^{195,42}$. However, the issues described above for conventional RNA-seq are still valid and can even be more acute. For example, the requirement for nuclear fractionation when analysing neurons with ScRNAseq leads to a large fraction of intronic reads and may obscure autonomous TE transcription.

The study of TE expression will also undoubtedly benefit from long-read single molecule sequencing technologies, such as those provided by PacBio or Oxford Nanopore ${ }^{196}$. Full-length RNA-seq could considerably reduce the proportion of ambiguously mapped reads, at least in theory, and could provide locus-specific expression levels. This strategy has the potential to reveal the nature of the expressed transcripts, including co-transcripts or chimeric transcripts. A first proof-of-principle was obtained in the migratory locust, Locusta migratoria, which possesses one of the largest sequenced genome $(6.5 \mathrm{~Gb})$. Full-length cDNA nanopore sequencing revealed a high proportion of exonized TEs in this organism ${ }^{197}$. PacBio sequencing of fairly long and bulk 5'-RACE products derived from L1 elements in human cell lines was also useful in facilitating the identification of loci producing $L 1$ full-length unit transcripts ${ }^{99}$.

Coupling whole-genome DNA sequencing and de novo assembly with full-length RNAseq can aid in taking into account sequence and insertional polymorphisms in TE transcriptomics studies. Indeed, long-read sequencing can significantly improve the detection of polymorphic TEs, particularly in low-complexity or repeated regions of the genome ${ }^{198,199}$. In addition, direct single-molecule sequencing can identify DNA modifications associated with the epigenetic regulation of $\mathrm{TEs}^{200-203}$. The promises of long-read sequencing are currently hampered by error rates that can far exceed the sequence divergence between TE loci. Thus, error correction methods, such as consensus-based error correction through rolling-circle amplification, tandem sequencing of both strands, or tagging with unique molecular identifiers, must be applied before these techniques can be employed successfully to study TE expression ${ }^{204,205}$.

\section{Conclusions}

Studies of TE transcription face three major difficulties: mappability, polymorphisms and transcript identity (Fig. 6). Clearly, some of these difficulties are also encountered with other sequencing approaches when studying TEs, and can be even more pronounced (discussed $\mathrm{in}^{42}$ ). For example, in bisulfite sequencing experiments to profile cytosine methylation, reads have reduced sequence complexity due to the chemical treatment, and are notoriously difficult to map to $\mathrm{TEs}^{42}$. Recent years have seen exciting advances in sequencing and computational approaches that were designed to specifically solve one or several of these challenges. These 
developments have boosted investigations into TE expression, shedding light on an entire new world of regulatory processes ${ }^{206}$. Nevertheless, none of the tools or approaches described here can bring a comprehensive solution on its own. Ultimately, the questions investigated should guide experimental design and subsequent analyses. Table 1 highlights the key features and limitations of different strategies. Integrating complementary methods or strategies, always in light of the specific aspect of TE biology that is being investigated, remains the best strategy for assessing and interpreting TE expression at the moment.

\section{References}

1. Chénais, B., Caruso, A., Hiard, S. \& Casse, N. The impact of transposable elements on eukaryotic genomes: From genome size increase to genetic adaptation to stressful environments. Gene 509, 7-15 (2012).

2. Lisch, D. How important are transposons for plant evolution? Nat. Rev. Genet. 14, 49-61 (2013).

3. Faulkner, G. J. \& Garcia-Perez, J. L. L1 Mosaicism in mammals: extent, effects, and evolution. Trends in Genetics 33, 802-816 (2017).

4. Chuong, E. B., Elde, N. C. \& Feschotte, C. Regulatory activities of transposable elements: from conflicts to benefits. Nat. Rev. Genet. 18, 71-86 (2017).

5. Payer, L. M. \& Burns, K. H. Transposable elements in human genetic disease. Nat. Rev. Genet. 20, 760-772 (2019).

6. Tam, O. H., Ostrow, L. W. \& Gale Hammell, M. Diseases of the nERVous system: retrotransposon activity in neurodegenerative disease. Mob. DNA 10, 32 (2019).

7. Sotero-Caio, C. G., Platt, R. N., II, Suh, A. \& Ray, D. A. Evolution and diversity of transposable elements in vertebrate genomes. Genome Biol. Evol. 9, 161-177 (2017).

8. Cho, J. \& Paszkowski, J. Regulation of rice root development by a retrotransposon acting as a microRNA sponge. elife 6, 796 (2017).

9. Brattås, P. L. et al. TRIM28 controls a gene regulatory network based on endogenous retroviruses in human neural progenitor cells. Cell Rep. 18, 1-11 (2017).

10. Petri, R. et al. LINE-2 transposable elements are a source of functional human microRNAs and target sites. PLoS Genet. 15, e1008036 (2019).

11. Kashkush, K., Feldman, M. \& Levy, A. A. Transcriptional activation of retrotransposons alters the expression of adjacent genes in wheat. Nat. Genet. 33, 102-106 (2003).

12. Percharde, M. et al. A LINE1-nucleolin partnership regulates early development and ESC identity. Cell 174, 391-405.e19 (2018).

13. Conte, C., Dastugue, B. \& Vaury, C. Promoter competition as a mechanism of transcriptional interference mediated by retrotransposons. EMBO J. 21, 3908-3916 (2002).

14. Jachowicz, J. W. et al. LINE-1 activation after fertilization regulates global chromatin accessibility in the early mouse embryo. Nat. Genet. 49, 1502-1510 (2017).

15. Stetson, D. B., Ko, J. S., Heidmann, T. \& Medzhitov, R. Trex1 prevents cell-intrinsic initiation 
of autoimmunity. Cell 134, 587-598 (2008).

16. Aravin, A. A. et al. Double-stranded RNA-mediated silencing of genomic tandem repeats and transposable elements in the D. melanogaster germline. Curr. Biol. 11, 1017-1027 (2001).

17. De Cecco, M. et al. L1 drives IFN in senescent cells and promotes age-associated inflammation. Nature 566, 73-78 (2019).

18. Goic, B. et al. RNA-mediated interference and reverse transcription control the persistence of RNA viruses in the insect model Drosophila. Nat. Immunol. 14, 396-403 (2013).

19. Bourgeois, Y. \& Boissinot, S. On the population dynamics of junk: a review on the population genomics of transposable elements. Genes 10, 419-423 (2019).

20. Khan, H., Smit, A. \& Boissinot, S. Molecular evolution and tempo of amplification of human LINE-1 retrotransposons since the origin of primates. Genome Res. 16, 78-87 (2006).

21. Huang, C. R. L., Burns, K. H. \& Boeke, J. D. Active transposition in genomes. Annu. Rev. Genet. 46, 651-675 (2012).

22. Mills, R. E., Bennett, E. A., Iskow, R. C. \& Devine, S. E. Which transposable elements are active in the human genome? Trends Genet. 23, 183-191 (2007).

23. Brouha, B. et al. Hot L1s account for the bulk of retrotransposition in the human population. Proc. Natl. Acad. Sci. U.S.A. 100, 5280-5285 (2003).

24. Beck, C. R. et al. LINE-1 retrotransposition activity in human genomes. Cell 141, 1159-1170 (2010).

25. Tubio, J. M. C. et al. Extensive transduction of nonrepetitive DNA mediated by L1 retrotransposition in cancer genomes. Science 345, 1251343-1251343 (2014).

Tubio et al. (2014), Gardner et al. (2017) and Rodriguez-Martin et al. (2020) identify progenitor L1 elements active in humans from whole genome sequencing using 3' transductions and internal SNPs in L1 sequences.

26. Gardner, E. J. et al. The Mobile Element Locator Tool (MELT): population-scale mobile element discovery and biology. Genome Res. 27, 1916-1929 (2017).

27. Rodriguez-Martin, B. et al. Pan-cancer analysis of whole genomes identifies driver rearrangements promoted by LINE-1 retrotransposition. Nat. Genet. 52, 306-319 (2020).

28. Deininger, P. L., Batzer, M. A., Hutchison, C. A. \& Edgell, M. H. Master genes in mammalian repetitive DNA amplification. Trends Genet. 8, 307-311 (1992).

29. Jacobs, F. M. J. et al. An evolutionary arms race between KRAB zinc-finger genes ZNF91/93 and SVA/L1 retrotransposons. Nature 516, 242-245 (2014).

30. Imbeault, M., Helleboid, P.-Y. \& Trono, D. KRAB zinc-finger proteins contribute to the evolution of gene regulatory networks. Nature 543, 550-554 (2017).

31. Sanchez-Luque, F. J. et al. LINE-1 evasion of epigenetic repression in humans. Mol. Cell 75, 590-604.e12 (2019).

Sanchez-Luque et al. (2019) and Seleme et al. (2006) show that a given L1 locus can exhibit internal sequence variation leading to differences of retrotransposition activity between individuals.

32. Boissinot, S., Entezam, A., Young, L., Munson, P. J. \& Furano, A. V. The insertional history 
of an active family of L1 retrotransposons in humans. Genome Res. 14, 1221-1231 (2004).

33. Scott, E. C. et al. A hot $L 1$ retrotransposon evades somatic repression and initiates human colorectal cancer. Genome Res. 26, 745-755 (2016).

This study resequenced all non-reference L1 elements in a colon cancer case to identify internal diagnostic SNPs and subsequently which elements are expressed in the sample.

34. Chalopin, D., Naville, M., Plard, F., Galiana, D. \& Volff, J.-N. Comparative analysis of transposable elements highlights mobilome diversity and evolution in vertebrates. Genome Biol. Evol. 7, 567-580 (2015).

35. Quadrana, L. et al. The Arabidopsis thaliana mobilome and its impact at the species level. eLife 5, e15716 (2016).

36. McCullers, T. J. \& Steiniger, M. Transposable elements in Drosophila. Mob Genet. Elements 7, 1-18 (2017).

37. Vitte, C. \& Panaud, O. LTR retrotransposons and flowering plant genome size: emergence of the increase/decrease model. Cytogenet Genome Res. 110, 91-107 (2005).

38. Hawkins, J. S., Proulx, S. R., Rapp, R. A. \& Wendel, J. F. Rapid DNA loss as a counterbalance to genome expansion through retrotransposon proliferation in plants. Proc. Natl. Acad. Sci. U.S.A. 106, 17811-17816 (2009).

39. Kapusta, A., Suh, A. \& Feschotte, C. Dynamics of genome size evolution in birds and mammals. Proc. Natl. Acad. Sci. U.S.A. 114, E1460-E1469 (2017).

Kapusta et al. (2017) and Kelley et al. (2012) discovered that a large fraction of longnon-coding RNA (IncRNA) derives from TEs in Vertebrates.

40. Goerner-Potvin, P. \& Bourque, G. Computational tools to unmask transposable elements. Nat. Rev. Genet. 19, 688-704 (2018).

41. Vendrell-Mir, P. et al. A benchmark of transposon insertion detection tools using real data. Mob. DNA 10, 53 (2019).

42. O'Neill, K., Brocks, D. \& Hammell, M. G. Mobile genomics: tools and techniques for tackling transposons. Philos. Trans. R. Soc. Lond. B. Biol. Sci. 375, 20190345 (2020).

43. Sudmant, P. H. et al. An integrated map of structural variation in 2,504 human genomes. Nature 526, 75-81 (2015).

44. Ewing, A. D. \& Kazazian, H. H. High-throughput sequencing reveals extensive variation in human-specific L1 content in individual human genomes. Genome Res. 20, 1262-1270 (2010).

45. Maksakova, I. A. et al. Retroviral elements and their hosts: insertional mutagenesis in the mouse germ line. PLoS Genet. 2, e2 (2006).

46. Zhang, Y., Maksakova, I. A., Gagnier, L., van de Lagemaat, L. N. \& Mager, D. L. Genomewide assessments reveal extremely high levels of polymorphism of two active families of mouse endogenous retroviral elements. PLoS Genet. 4, e1000007 (2008).

47. Nellåker, C. et al. The genomic landscape shaped by selection on transposable elements across 18 mouse strains. Genome Biol. 13, R45 (2012). 
48. Richardson, S. R. et al. Heritable L1 retrotransposition in the mouse primordial germline and early embryo. Genome Res. 27, 1395-1405 (2017).

49. Carpentier, M.-C. et al. Retrotranspositional landscape of Asian rice revealed by 3000 genomes. Nat. Commun. 10, 24 (2019).

50. Feusier, J. et al. Pedigree-based estimation of human mobile element retrotransposition rates. Genome Res. 29, 1567-1577 (2019).

51. Rech, G. E. et al. Stress response, behavior, and development are shaped by transposable element-induced mutations in Drosophila. PLoS Genet. 15, e1007900 (2019).

52. González, J., Karasov, T. L., Messer, P. W. \& Petrov, D. A. Genome-wide patterns of adaptation to temperate environments associated with transposable elements in Drosophila. PLoS Genet. 6, e1000905 (2010).

53. Payer, L. M. et al. Structural variants caused by Alu insertions are associated with risks for many human diseases. Proc. Natl. Acad. Sci. U.S.A. 114, E3984-E3992 (2017).

54. Kazazian, H. H., Jr. \& Moran, J. V. Mobile DNA in health and disease. N. Engl. J. Med. 377, 361-370 (2017).

55. Seleme, M. D. C. et al. Extensive individual variation in L1 retrotransposition capability contributes to human genetic diversity. Proc. Natl. Acad. Sci. U.S.A. 103, 6611-6616 (2006).

56. Swergold, G. D. Identification, characterization, and cell specificity of a human LINE-1 promoter. Mol. Cell. Biol. 10, 6718-6729 (1990).

57. Thompson, P. J., Macfarlan, T. S. \& Lorincz, M. C. Long terminal repeats: from parasitic elements to building blocks of the transcriptional regulatory repertoire. Mol. Cell 62, 766-776 (2016).

58. Mighell, A. J., Markham, A. F. \& Robinson, P. A. Alu sequences. FEBS Lett. 417, 1-5 (1997).

59. Hancks, D. C., Ewing, A. D., Chen, J. E., Tokunaga, K. \& Kazazian, H. H. Exon-trapping mediated by the human retrotransposon SVA. Genome Res. 19, 1983-1991 (2009).

60. Honigman, A., Bar-Shira, A., Silberberg, H. \& Panet, A. Generation of a uniform 3' end RNA of murine leukemia virus. J. Virol. 53, 330-334 (1985).

61. Dombroski, B. A., Mathias, S. L., Nanthakumar, E., Scott, A. F. \& Kazazian, H. H. Isolation of an active human transposable element. Science 254, 1805-1808 (1991).

62. Conti, A. et al. Identification of RNA polymerase III-transcribed Alu loci by computational screening of RNA-Seq data. Nucleic Acids Res. 43, 817-835 (2014).

63. Holmes, S. E., Dombroski, B. A., Krebs, C. M., Boehm, C. D. \& Kazazian, H. H. A new retrotransposable human L1 element from the LRE2 locus on chromosome 1q produces a chimaeric insertion. Nat. Genet. 7, 143-148 (1994).

64. Moran, J. V., DeBerardinis, R. J. \& Kazazian, H. H. Exon shuffling by L1 retrotransposition. Science 283, 1530-1534 (1999).

65. McKerrow, W. \& Fenyö, D. L1EM: A tool for accurate locus specific LINE-1 RNA quantification. Bioinformatics 544, 115 (2019).

66. Pickeral, O. K., Makałowski, W., Boguski, M. S. \& Boeke, J. D. Frequent human genomic DNA transduction driven by LINE-1 retrotransposition. Genome Res. 10, 411-415 (2000). 
67. Goodier, J. L., Ostertag, E. M. \& Kazazian, H. H. Transduction of 3'-flanking sequences is common in L1 retrotransposition. Hum. Mol. Genet. 9, 653-657 (2000).

68. Lander, E. S. et al. Initial sequencing and analysis of the human genome. Nature 409, 860921 (2001).

69. Evrony, G. D. et al. Single-neuron sequencing analysis of L1 retrotransposition and somatic mutation in the human brain. Cell 151, 483-496 (2012).

70. Damert, A. et al. 5'-Transducing SVA retrotransposon groups spread efficiently throughout the human genome. Genome Res. 19, 1992-2008 (2009).

71. Eickbush, D. G. \& Eickbush, T. H. R2 retrotransposons encode a self-cleaving ribozyme for processing from an rRNA cotranscript. Mol. Cell. Biol. 30, 3142-3150 (2010).

72. Perepelitsa-Belancio, V. \& Deininger, P. RNA truncation by premature polyadenylation attenuates human mobile element activity. Nat. Genet. 35, 363-366 (2003).

73. Schrom, E.-M., Moschall, R., Schuch, A. \& Bodem, J. Regulation of retroviral polyadenylation. Adv. Virus Res. 85, 1-24 (2013).

74. Belancio, V. P., Hedges, D. J. \& Deininger, P. LINE-1 RNA splicing and influences on mammalian gene expression. Nucleic Acids Res. 34, 1512-1521 (2006).

75. Teixeira, F. K. et al. PiRNA-mediated regulation of transposon alternative splicing in the soma and germ line. Nature 552, 268-272 (2017).

76. Kines, K. J., Sokolowski, M., DeHaro, D. L., Christian, C. M. \& Belancio, V. P. Potential for genomic instability associated with retrotranspositionally-incompetent L1 loci. Nucleic Acids Res. 42, 10488-10502 (2014).

77. Saha, A. et al. A trans-dominant form of Gag restricts Ty1 retrotransposition and mediates copy number control. J. Virol. 89, 3922-3938 (2015).

78. Speek, M. Antisense promoter of human L1 retrotransposon drives transcription of adjacent cellular genes. Mol. Cell. Biol. 21, 1973-1985 (2001).

79. Cruickshanks, H. A. \& Tufarelli, C. Isolation of cancer-specific chimeric transcripts induced by hypomethylation of the LINE-1 antisense promoter. Genomics 94, 397-406 (2009).

80. Weber, B., Kimhi, S., Howard, G., Eden, A. \& Lyko, F. Demethylation of a LINE-1 antisense promoter in the cMet locus impairs Met signalling through induction of illegitimate transcription. Oncogene 29, 5775-5784 (2010).

81. $\mathrm{Li}$, J. et al. An antisense promoter in mouse $\mathrm{L} 1$ retrotransposon open reading frame-1 initiates expression of diverse fusion transcripts and limits retrotransposition. Nucleic Acids Res. 42, 4546-4562 (2014).

82. Denli, A. M. et al. Primate-Specific ORF0 Contributes to Retrotransposon-Mediated Diversity. Cell 163, 583-593 (2015).

The first study to use mass-spectrometry data on a large-scale to identify unknown TE chimeric proteins.

83. Russo, J., Harrington, A. W. \& Steiniger, M. Antisense Transcription of Retrotransposons in Drosophila: An Origin of Endogenous Small Interfering RNA Precursors. Genetics 202, 107121 (2016). 
84. Harrington, A. W. \& Steiniger, M. Bioinformatic analyses of sense and antisense expression from terminal inverted repeat transposons in Drosophila somatic cells. FLY 10, 1-10 (2016).

85. Zingler, N. et al. Analysis of 5 junctions of human LINE-1 and Alu retrotransposons suggests an alternative model for 5'-end attachment requiring microhomology-mediated end-joining. Genome Res. 15, 780-789 (2005).

86. Suzuki, J. et al. Genetic evidence that the non-homologous end-joining repair pathway is involved in LINE retrotransposition. PLoS Genet. 5, e1000461 (2009).

87. Larson, P. A. et al. Spliced integrated retrotransposed element (SpIRE) formation in the human genome. PLoS Biol. 16, e2003067 (2018).

88. Penzkofer, T. et al. L1Base 2 - more retrotransposition-active LINE-1s, more mammalian genomes. Nucleic Acids Res. 45, D68-D73 (2017).

89. Wirth, T., Glöggler, K., Baumruker, T., Schmidt, M. \& Horak, I. Family of middle repetitive DNA sequences in the mouse genome with structural features of solitary retroviral long terminal repeats. Proc. Natl. Acad. Sci. U.S.A. 80, 3327-3330 (1983).

90. Mager, D. L. \& Goodchild, N. L. Homologous recombination between the LTRs of a human retrovirus-like element causes a 5-kb deletion in two siblings. Am. J. Hum. Genet.. 45, 848854 (1989).

91. Vitte, C. \& Panaud, O. Formation of solo-LTRs through unequal homologous recombination counterbalances amplifications of LTR retrotransposons in rice Oryza sativa L. Mol. Biol.

Evol. 20, 528-540 (2003).

92. Cossu, R. M. et al. LTR Retrotransposons show low levels of unequal recombination and high rates of intraelement gene conversion in large plant genomes. Genome Biol. Evol. 9, 3449-3462 (2017).

93. Rebollo, R., Farivar, S. \& Mager, D. L. C-GATE - catalogue of genes affected by transposable elements. Mob. DNA 3, 9 (2012).

94. Kelley, D. \& Rinn, J. Transposable elements reveal a stem cell-specific class of long noncoding RNAs. Genome Biol. 13, R107 (2012).

95. Kapusta, A. et al. Transposable elements are major contributors to the origin, diversification, and regulation of vertebrate long noncoding RNAs. PLoS Genet. 9, e1003470 (2013).

96. Lu, X. et al. The retrovirus HERVH is a long noncoding RNA required for human embryonic stem cell identity. Nat. Struct. Mol. Biol. 21, 423-425 (2014).

97. Wang, J. et al. Primate-specific endogenous retrovirus-driven transcription defines naive-like stem cells. Nature 516, 405-409 (2014).

98. Izsvák, Z., Wang, J., Singh, M., Mager, D. L. \& Hurst, L. D. Pluripotency and the endogenous retrovirus HERVH: conflict or serendipity? BioEssays 38, 109-117 (2015).

99. Deininger, P. et al. A comprehensive approach to expression of L1 loci. Nucleic Acids Res. 45, e31 (2017).

100. Navarro, F. C. P. et al. TeXP: Deconvolving the effects of pervasive and autonomous transcription of transposable elements. PLoS Comput. Biol. 15, e1007293 (2019).

101. Jensen, T. H., Jacquier, A. \& Libri, D. Dealing with pervasive transcription. Mol. Cell 52, 473- 
484 (2013).

102. Lee, H., Zhang, Z. \& Krause, H. M. Long noncoding RNAs and repetitive elements: junk or intimate evolutionary partners? Trends Genet. 35, 892-902 (2019).

103. Kim, T.-K., Hemberg, M. \& Gray, J. M. Enhancer RNAs: a class of long noncoding RNAs synthesized at enhancers. Cold Spring Harb Perspect Biol 7, a018622 (2015).

104. Wassenegger, M., Heimes, S., Riedel, L. \& Sänger, H. L. RNA-directed de novo methylation of genomic sequences in plants. Cell 76, 567-576 (1994).

105. Fire, A. et al. Potent and specific genetic interference by double-stranded RNA in Caenorhabditis elegans. Nature 391, 806-811 (1998).

106. Yang, N. \& Kazazian, H. H. L1 retrotransposition is suppressed by endogenously encoded small interfering RNAs in human cultured cells. Nat. Struct. Mol. Biol. 13, 763-771 (2006).

107. Slotkin, R. K. et al. Epigenetic reprogramming and small RNA silencing of transposable elements in pollen. Cell 136, 1451-1454 (2009).

108. Heras, S. R. et al. The Microprocessor controls the activity of mammalian retrotransposons. Nat. Struct. Mol. Biol. 20, 1173-1181 (2013).

109. Cuerda-Gil, D. \& Slotkin, R. K. Non-canonical RNA-directed DNA methylation. Nat. Plants2, 567-8 (2016).

110. van de Lagemaat, L. N., Medstrand, P. \& Mager, D. L. Multiple effects govern endogenous retrovirus survival patterns in human gene introns. Genome Biol. 7, R86 (2006).

111. Berrens, R. V. et al. An endosiRNA-based repression mechanism counteracts transposon activation during global DNA demethylation in embryonic stem cells. Stem Cell 21, 694703.e7 (2017).

112. Gong, C., Tang, Y. \& Maquat, L. E. mRNA-mRNA duplexes that autoelicit Staufen1-mediated mRNA decay. Nat. Struct. Mol. Biol. 20, 1214-1222 (2013).

113. Roulois, D. et al. DNA-demethylating agents target colorectal cancer cells by inducing viral mimicry by endogenous transcripts. Cell 162, 961-973 (2015).

Roulois et al. (2015), Chiappinelli et al. (2015) and Brocks et al. (2016) reveal mechanisms by which the reactivation of transposable elements with drugs targeting epigenetic pathway can kill cancer cells.

114. Chiappinelli, K. B. et al. Inhibiting DNA methylation causes an interferon response in cancer via dsRNA including endogenous retroviruses. Cell 162, 974-986 (2015).

115. Skowronski, J. \& Singer, M. F. Expression of a cytoplasmic LINE-1 transcript is regulated in a human teratocarcinoma cell line. Proc. Natl. Acad. Sci. U.S.A. 82, 6050-6054 (1985).

116. Belancio, V. P., Roy-Engel, A. M., Pochampally, R. R. \& Deininger, P. Somatic expression of LINE-1 elements in human tissues. Nucleic Acids Res. 38, 3909-3922 (2010).

Together with Deininger et al. (2017), this work shows that the majority of L1 RNA detected in somatic cells is not unit-length RNA but rather truncated L1 RNA or derives from co-transcription or pervasive transcription.

117. Morillon, A., Bénard, L., Springer, M. \& Lesage, P. Differential effects of chromatin and Gcn4 on the 50 -fold range of expression among individual yeast Ty1 retrotransposons. Mol. Cell. 
Biol. 22, 2078-2088

118. Slotkin, R. K. \& Martienssen, R. Transposable elements and the epigenetic regulation of the genome. Nat. Rev. Genet. 8, 272-285 (2007).

119. Pizarro, J. G. \& Cristofari, G. Post-transcriptional control of LINE-1 retrotransposition by cellular host factors in somatic cells. Front. Cell Dev. Biol. 4, 14 (2016).

120. Goodier, J. L. Restricting retrotransposons: a review. Mob. DNA 7, 344 (2016).

121. Schorn, A. J., Gutbrod, M. J., LeBlanc, C. \& Martienssen, R. LTR-retrotransposon control by tRNA-derived small RNAs. Cell 170, 61-71.e11 (2017).

122. Hohjoh, H. \& Singer, M. F. Cytoplasmic ribonucleoprotein complexes containing human LINE-1 protein and RNA. EMBO J. 15, 630-639 (1996).

123. Biczysko, W., Pienkowski, M., Solter, D. \& Koprowski, H. Virus particles in early mouse embryos. J. Natl. Cancer Inst. 51, 1041-1050 (1973).

124. Kulpa, D. A. \& Moran, J. V. Ribonucleoprotein particle formation is necessary but not sufficient for LINE-1 retrotransposition. Hum. Mol. Genet. 14, 3237-3248 (2005).

125. Grow, E. J. et al. Intrinsic retroviral reactivation in human preimplantation embryos and pluripotent cells. Nature 522, 221-225 (2015).

126. Seifarth, W. et al. Comprehensive analysis of human endogenous retrovirus transcriptional activity in human tissues with a retrovirus-specific microarray. J. Virol. 79, 341-352 (2005).

127. Picault, N. et al. Identification of an active LTR retrotransposon in rice. Plant J. 58, 754-765 (2009).

128. Horard, B. et al. Global analysis of DNA methylation and transcription of human repetitive sequences. Epigenetics 4, 339-350 (2009).

129. Reichmann, J. et al. Microarray analysis of LTR retrotransposon silencing identifies Hdac1 as a regulator of retrotransposon expression in mouse embryonic stem cells. PLoS Comput.

Biol. 8, e1002486 (2012).

130. Gnanakkan, V. P. et al. TE-array--a high throughput tool to study transposon transcription. BMC Genomics 14, 869 (2013).

131. Faulkner, G. J. et al. A rescue strategy for multimapping short sequence tags refines surveys of transcriptional activity by CAGE. Genomics 91, 281-288 (2008).

132. Chung, N. et al. Transcriptome analyses of tumor-adjacent somatic tissues reveal genes coexpressed with transposable elements. Mob. DNA 10, 15 (2019).

Chung et al. (2019) and McKerrow et al. (2019) both propose strategies based on the EM-algorithm to discriminate and quantify TE transcript types.

133. Sexton, C. E. \& Han, M. V. Paired-end mappability of transposable elements in the human genome. Mob. DNA 10, 29 (2019).

134. Teissandier, A., Servant, N., Barillot, E. \& Bourc'his, D. Tools and best practices for retrotransposon analysis using high-throughput sequencing data. Mob. DNA 10, 52 (2019).

135. Bao, W., Kojima, K. K. \& Kohany, O. Repbase Update, a database of repetitive elements in eukaryotic genomes. Mob. DNA 6, 11 (2015).

136. Lerat, E., Fablet, M., Modolo, L., Lopez-Maestre, H. \& Vieira, C. TEtools facilitates big data 
expression analysis of transposable elements and reveals an antagonism between their activity and that of piRNA genes. Nucleic Acids Res. 45, 1-12 (2017).

137. Romero-Soriano, V. et al. Transposable element misregulation is linked to the divergence between parental piRNA pathways in Drosophila hybrids. Genome Biol. Evol. 9, 1450-1470 (2017).

138. Zeng, Z. et al. Genome-wide DNA methylation and transcriptomic profiles in the lifestyle strategies and asexual development of the forest fungal pathogen Heterobasidion parviporum. Epigenetics 14, 16-40 (2019).

139. Song, H. et al. Rapid evolution of piRNA pathway and its transposon targets in Japanese flounder (Paralichthys olivaceus). Comp. Biochem. Physiol. Part D Genomics Proteomics 31, 100609 (2019).

140. Langmead, B. \& Salzberg, S. L. Fast gapped-read alignment with Bowtie 2. Nat. Methods 9, 357-359 (2012).

141. Li, H. \& Durbin, R. Fast and accurate short read alignment with Burrows-Wheeler transform. Bioinformatics 25, 1754-1760 (2009).

142. Trapnell, C., Pachter, L. \& Salzberg, S. L. TopHat: discovering splice junctions with RNASeq. Bioinformatics 25, 1105-1111 (2009).

143. Dobin, A. et al. STAR - ultrafast universal RNA-seq aligner. Bioinformatics 29, 15-21 (2013).

144. Criscione, S. W., Zhang, Y., Thompson, W., Sedivy, J. M. \& Neretti, N. Transcriptional landscape of repetitive elements in normal and cancer human cells. BMC Genomics 15, 583 (2014).

145. Yang, W. R., Ardeljan, D., Pacyna, C. N., Payer, L. M. \& Burns, K. H. SQuIRE reveals locusspecific regulation of interspersed repeat expression. Nucleic Acids Res. 47, e27 (2019).

146. Valdebenito-Maturana, B. \& Riadi, G. TEcandidates: prediction of genomic origin of expressed transposable elements using RNA-seq data. Bioinformatics 34, 3915-3916 (2018).

147. Li, B. \& Dewey, C. N. RSEM: accurate transcript quantification from RNA-Seq data with or without a reference genome. BMC Bioinformatics 12, 323 (2011).

148. Jin, Y., Tam, O. H., Paniagua, E. \& Hammell, M. TEtranscripts: a package for including transposable elements in differential expression analysis of RNA-seq datasets.

Bioinformatics 31, 3593-3599 (2015).

TEtranscripts is the first application of the EM-algorithm to TE RNA-seq analyses, and one of the most popular software packages dedicated to this task since its release.

149. Bendall, M. L. et al. Telescope: characterization of the retrotranscriptome by accurate estimation of transposable element expression. PLoS Comput. Biol. 15, e1006453 (2019).

150. Bray, N. L., Pimentel, H., Melsted, P. \& Pachter, L. Near-optimal probabilistic RNA-seq quantification. Nat. Biotechnol. 34, 525-527 (2016).

151. Patro, R., Duggal, G., Love, M. I., Irizarry, R. A. \& Kingsford, C. Salmon provides fast and bias-aware quantification of transcript expression. Nat. Methods 14, 417-419 (2017).

152. Jeong, H.-H., Yalamanchili, H. K., Guo, C., Shulman, J. M. \& Liu, Z. An ultra-fast and 
scalable quantification pipeline for transposable elements from next generation sequencing data. Pac Symp Biocomput 23, 168-179 (2018).

153. Kong, Y. et al. Transposable element expression in tumors is associated with immune infiltration and increased antigenicity. Nat. Commun. 10, 5228 (2019).

154. Philippe, C. et al. Activation of individual L1 retrotransposon instances is restricted to celltype dependent permissive loci. eLife 5, 166 (2016).

This study proposes the first strategy to profile the expression of reference and nonreference $L 1$ elements at the locus level by integrating targeted resequencing of L1 elements (ATLAS-seq), RNA-seq and ChIP-seq data.

155. Ewing, A. D. Transposable element detection from whole genome sequence data. Mob. DNA 6, 24 (2015).

156. Mir, A. A., Philippe, C. \& Cristofari, G. euL1db: the European database of L1HS retrotransposon insertions in humans. Nucleic Acids Res. 43, D43-D47 (2015).

157. Tokuyama, M. et al. ERVmap analysis reveals genome-wide transcription of human endogenous retroviruses. Proc. Natl. Acad. Sci. U.S.A. 115, 12565-12572 (2018).

158. Ansaloni, F., Scarpato, M., Di Schiavi, E., Gustincich, S. \& Sanges, R. Exploratory analysis of transposable elements expression in the C. elegans early embryo. BMC Bioinformatics 20, 484 (2019).

159. Kaul, T., Morales, M. E., Sartor, A. O., Belancio, V. P. \& Deininger, P. Comparative analysis on the expression of L1 loci using various RNA-Seq preparations. Mob. DNA 11, 860 (2020).

160. Faulkner, G. J. et al. The regulated retrotransposon transcriptome of mammalian cells. Nat. Genet. 41, 563-571 (2009).

This article offers the first genome-wide description of TE transcription across multiple tissues using CAGE data from the PHANTOM project.

161. Brocks, D. et al. DNMT and HDAC inhibitors induce cryptic transcription start sites encoded in long terminal repeats. Nat. Genet. 49, 1052-1060 (2017).

162. Batut, P., Dobin, A., Plessy, C., Carninci, P. \& Gingeras, T. R. High-fidelity promoter profiling reveals widespread alternative promoter usage and transposon-driven developmental gene expression. Genome Res. 23, 169-180 (2013).

163. Rangwala, S. H., Zhang, L. \& Kazazian, H. H. Many LINE1 elements contribute to the transcriptome of human somatic cells. Genome Biol. 10, R100 (2009).

164. Macia, A. et al. Epigenetic control of retrotransposon expression in human embryonic stem cells. Mol. Cell. Biol. 31, 300-316 (2011).

165. Lock, F. E. et al. Distinct isoform of FABP7 revealed by screening for retroelement-activated genes in diffuse large B-cell lymphoma. Proc. Natl. Acad. Sci. U.S.A. 111, E3534-E3543 (2014).

166. Morgan, H. D., Sutherland, H. G., Martin, D. I. \& Whitelaw, E. Epigenetic inheritance at the agouti locus in the mouse. Nat. Genet. 23, 314-318 (1999).

167. Wheelan, S. J., Aizawa, Y., Han, J. S. \& Boeke, J. D. Gene-breaking: a new paradigm for human retrotransposon-mediated gene evolution. Genome Res. 15, 1073-1078 (2005). 
168. Shen, S. et al. Widespread establishment and regulatory impact of Alu exons in human genes. Proc. Natl. Acad. Sci. U.S.A. 108, 2837-2842 (2011).

169. Butelli, E. et al. Retrotransposons control fruit-specific, cold-dependent accumulation of anthocyanins in blood oranges. The Plant Cell 24, 1242-1255 (2012).

170. Ong-Abdullah, M. et al. Loss of Karma transposon methylation underlies the mantled somaclonal variant of oil palm. Nature 525, 533-537 (2015).

171. Barau, J. et al. The DNA methyltransferase DNMT3C protects male germ cells from transposon activity. Science 354, 909-912 (2016).

172. Attig, J. et al. LTR retroelement expansion of the human cancer transcriptome and immunopeptidome revealed by de novo transcript assembly. Genome Res. 29, 1578-1590 (2019).

173. Jang, H. S. et al. Transposable elements drive widespread expression of oncogenes in human cancers. Nat. Genet. 51, 611-617 (2019).

Attig et al. (2019) and Jang et al. (2019) provides a systematic view of tumor-specific transcripts and antigens derived from TEs.

174. Nigumann, P., Redik, K., Mätlik, K. \& Speek, M. Many human genes are transcribed from the antisense promoter of L1 retrotransposon. Genomics 79, 628-634 (2002).

175. Peaston, A. E. et al. Retrotransposons regulate host genes in mouse oocytes and preimplantation embryos. Dev. Cell 7, 597-606 (2004).

176. Lipatov, M., Lenkov, K., Petrov, D. A. \& Bergman, C. M. Paucity of chimeric genetransposable element transcripts in the Drosophila melanogaster genome. BMC Biol. 3, 24 (2005).

177. Ha, H.-S. et al. Identification and characterization of transposable element-mediated chimeric transcripts from porcine Refseq and EST databases. Genes Genom. 34, 409-414 (2012).

178. Criscione, S. W. et al. Genome-wide characterization of human L1 antisense promoter-driven transcripts. BMC Genomics 17, 463 (2016).

179. Pinson, M.-E., Pogorelcnik, R., Court, F., Arnaud, P. \& Vaurs-Barrière, C. CLIFinder: identification of LINE-1 chimeric transcripts in RNA-seq data. Bioinformatics 34, 688-690 (2017).

180. Babaian, A. et al. LIONS: analysis suite for detecting and quantifying transposable element initiated transcription from RNA-seq. Bioinformatics 35, 3839-3841 (2019).

181. Wang, T. et al. A novel analytical strategy to identify fusion transcripts between repetitive elements and protein coding-exons using RNA-Seq. PLoS ONE 11, e0159028 (2016).

182. Larrosa, R., Arroyo, M., Bautista, R., López-Rodríguez, C. M. \& Claros, M. G. NearTrans can identify correlated expression changes between retrotransposons and surrounding genes in human cancer. Bioinformatics and Biomedical Engineering 10813, 373-382 (2018).

183. Karakülah, G., Arslan, N., Yandin, C. \& Suner, A. TEffectR: An R package for studying the potential effects of transposable elements on gene expression with linear regression model. PeerJ 7, e8192 (2019).

184. Decker, C. J. et al. dsRNA-Seq: identification of viral infection by purifying and sequencing 
dsRNA. Viruses 11, 943 (2019).

185. Castel, S. E. \& Martienssen, R. A. RNA interference in the nucleus: roles for small RNAs in transcription, epigenetics and beyond. Nat. Rev. Genet. 14, 100-112 (2013).

186. Johnson, N. R., Yeoh, J. M., Coruh, C. \& Axtell, M. J. Improved placement of multi-mapping small RNAs. G3 6, 2103-2111 (2016).

187. Bousios, A., Gaut, B. S. \& Darzentas, N. Considerations and complications of mapping small RNA high-throughput data to transposable elements. Mob. DNA 8, 3 (2017).

188. Garrison, E. et al. Variation graph toolkit improves read mapping by representing genetic variation in the reference. Nat. Biotechnol. 36, 875-879 (2018).

189. Rakocevic, G. et al. Fast and accurate genomic analyses using genome graphs. Nat. Genet. 51, 354-362 (2019).

190. Kim, D., Paggi, J. M., Park, C., Bennett, C. \& Salzberg, S. L. Graph-based genome alignment and genotyping with HISAT2 and HISAT-genotype. Nat. Biotechnol. 37, 907-915 (2019).

191. Sherman, R. M. \& Salzberg, S. L. Pan-genomics in the human genome era. Nat. Rev. Genet. 21, 243-254 (2020).

192. Maringer, K. et al. Proteomics informed by transcriptomics for characterising active transposable elements and genome annotation in Aedes aegypti. BMC Genomics 18, 101 (2017).

193. Davidson, A. D., Matthews, D. A. \& Maringer, K. Proteomics technique opens new frontiers in mobilome research. Mob Genet. Elements 7, 1-9 (2017).

194. Ardeljan, D. et al. LINE-1 ORF2p expression is nearly imperceptible in human cancers. Mob. DNA 11, 1-19 (2019).

195. Brocks, D., Chomsky, E., Mukamel, Z., Lifshitz, A. \& Tanay, A. Single cell analysis reveals dynamics of transposable element transcription following epigenetic de-repression. BioRxiv 462853 (2019)

196. Shahid, S. \& Slotkin, R. K. The current revolution in transposable element biology enabled by long reads. Curr. Opin. Genet. Dev. 54, 49-56 (2020).

197. Jiang, F. et al. Long-read direct RNA sequencing by 5'-Cap capturing reveals the impact of Piwi on the widespread exonization of transposable elements in locusts. RNA Biol. 16, 950959 (2019).

This study provides the first use of direct RNA sequencing by the Oxford Nanopore technology to characterize the impact of TE transcription on the transcriptome of a non-model organism.

198. Debladis, E., Llauro, C., Carpentier, M.-C., Mirouze, M. \& Panaud, O. Detection of active transposable elements in Arabidopsis thaliana using Oxford Nanopore Sequencing technology. BMC Genomics 18, 537 (2017).

199. Zhou, W. et al. Identification and characterization of occult human-specific LINE-1 insertions using long-read sequencing technology. Nucleic Acids Res. 409, 860 (2019).

200. Wu, T. P. et al. DNA methylation on N6-adenine in mammalian embryonic stem cells. Nature 532, 329-333 (2016). 
201. Simpson, J. T. et al. Detecting DNA cytosine methylation using nanopore sequencing. Nat. Methods 14, 407-410 (2017).

202. Liu, Q. et al. Detection of DNA base modifications by deep recurrent neural network on Oxford Nanopore sequencing data. Nat. Commun. 10, 2449 (2019).

203. Liu, Q., Georgieva, D. C., Egli, D. \& Wang, K. NanoMod: A computational tool to detect DNA modifications using Nanopore long-read sequencing data. BMC Genomics 20, 78 (2019).

204. Kutter, C., Jern, P. \& Suh, A. Bridging gaps in transposable element research with singlemolecule and single-cell technologies. Mob. DNA 9, 34 (2018).

205. Salk, J. J., Schmitt, M. W. \& Loeb, L. A. Enhancing the accuracy of next-generation sequencing for detecting rare and subclonal mutations. Nat. Rev. Genet. 19, 269-285 (2018).

206. Slotkin, R. K. The case for not masking away repetitive DNA. Mob. DNA 9, 15 (2018).

207. Finnegan, D. J. Eukaryotic transposable elements and genome evolution. Trends Genet. 5, 103-107 (1989).

208. Wicker, T. et al. A unified classification system for eukaryotic transposable elements. Nat. Rev. Genet. 8, 973-982 (2007).

209. Piégu, B., Bire, S., Arensburger, P. \& Bigot, Y. A survey of transposable element classification systems--a call for a fundamental update to meet the challenge of their diversity and complexity. Mol. Phylogenet. Evol. 86, 90-109 (2015).

210. Curcio, M. J. \& Derbyshire, K. M. The outs and ins of transposition: from mu to kangaroo. Nat. Rev. Mol. Cell Biol. 4, 865-877 (2003).

211. Hubley, R. et al. The Dfam database of repetitive DNA families. Nucleic Acids Res. 44, D81D89 (2016).

212. Amselem, J. et al. RepetDB: A unified resource for transposable element references. Mob. DNA 10, 6-8 (2019).

213. Herquel, B. et al. Trim24-repressed VL30 retrotransposons regulate gene expression by producing noncoding RNA. Nat. Struct. Mol. Biol. 20, 339-346 (2013).

214. Fadloun, A. et al. Chromatin signatures and retrotransposon profiling in mouse embryos reveal regulation of LINE-1 by RNA. Nat. Struct. Mol. Biol. 20, 332-338 (2013).

215. Derrien, T. et al. Fast computation and applications of genome mappability. PLoS ONE 7, e30377 (2012).

216. Karimzadeh, M., Ernst, C., Kundaje, A. \& Hoffman, M. M. Umap and Bismap: quantifying genome and methylome mappability. Nucleic Acids Res. 46, e120 (2018).

\section{Acknowledgements}

The authors apologize to the many colleagues who have made significant contributions to the field but whose work could not be cited or discussed owing to space limitations. The authors are grateful to P.A. Defossez and A. Doucet for critical reading of the manuscript. This work was supported by grants to G.C. from the Fondation pour la Recherche Médicale (FRM, 
DEQ20180339170), the Agence Nationale de la Recherche (LABEX SIGNALIFE, ANR-11LABX-0028-01; RetroMet, ANR-16-CE12-0020; ImpacTE, ANR-19-CE12-0032), the Canceropôle Provence-Alpes-Côte d'Azur, the French National Cancer Institute (INCa) and the Provence-Alpes-Côte d'Azur Region, CNRS (GDR 3546), and the University Hospital Federation (FHU) OncoAge.

\section{Author contributions}

The authors contributed equally to all aspects of the article.

\section{Competing interests}

S. L. declares no competing interests. G. C. is an unpaid associate editor of the journal Mobile DNA (Springer Nature).

\section{Publisher's note}

Springer Nature remains neutral with regard to jurisdictional claims in published maps and institutional affiliations.

\section{Peer review information}

Nature Reviews Genetics thanks G. J. Faulkner and the other, anonymous, reviewer(s) for their contribution to the peer review of this work. 
TABLE 1. Computational tools and approaches to analyse TE unit expression from RNAseq data.

\begin{tabular}{|c|c|c|c|c|c|c|c|}
\hline $\begin{array}{c}\text { Tools or } \\
\text { approaches }\end{array}$ & $\begin{array}{l}\text { Mapping or } \\
\text { pseudo- } \\
\text { mapping to }\end{array}$ & $\begin{array}{c}\text { Fate of } \\
\text { multimappers }\end{array}$ & $\begin{array}{c}\text { Type of } \\
\text { quantification } \\
\text { (F or } L)\end{array}$ & $\begin{array}{l}\text { Distinguishes } \\
\text { unit-length } \\
\text { transcripts } \\
\text { from other } \\
\text { TE-derived } \\
\text { transcripts }\end{array}$ & $\begin{array}{c}\text { Includes } \\
\text { polymorphic } \\
\text { TE } \\
\text { expression }\end{array}$ & Notes & Ref. \\
\hline TEtools & $\begin{array}{l}\text { TE pseudo- } \\
\text { genome }\end{array}$ & $\begin{array}{l}\text { randomly } \\
\text { assigned }\end{array}$ & $F$ & - & - & $\begin{array}{l}\text { applicable to } \\
\text { unassembled } \\
\text { genomes }\end{array}$ & 136 \\
\hline SalmonTE & $\begin{array}{l}\text { Consensus } \\
\text { transcriptome }\end{array}$ & EM algorithm & $\mathrm{F}$ & - & - & $\begin{array}{l}\text { fast pseudo- } \\
\text { mapping }\end{array}$ & 152 \\
\hline REdiscoverTE & $\begin{array}{c}\text { Model } \\
\text { transcriptome }\end{array}$ & EM algorithm & $F$ & + & - & $\begin{array}{l}\text { uses SalmonTE } \\
\text { algorithm }\end{array}$ & 153 \\
\hline TEtranscripts & $\begin{array}{l}\text { Reference } \\
\text { genome }\end{array}$ & EM algorithm & $\mathrm{F}$ & - & - & $\begin{array}{c}\text { one of the most } \\
\text { used tools, tested } \\
\text { on a wide variety } \\
\text { of organisms }\end{array}$ & 148 \\
\hline RepEnrich & $\begin{array}{l}\text { Reference } \\
\text { genome }\end{array}$ & $\begin{array}{l}\text { remapped on } \\
\text { TE pseudo- } \\
\text { genome }\end{array}$ & $\mathrm{F}$ & - & - & - & 144 \\
\hline TeXP & $\begin{array}{l}\text { Reference } \\
\text { genome }\end{array}$ & $\begin{array}{l}\text { randomly } \\
\text { assigned }\end{array}$ & $F$ & $+/-$ & - & $\begin{array}{c}\text { subtracts } \\
\text { pervasive } \\
\text { transcription but } \\
\text { not other forms of } \\
\text { chimeric } \\
\text { transcripts } \\
\end{array}$ & 100 \\
\hline ERVmap & $\begin{array}{l}\text { Reference } \\
\text { genome }\end{array}$ & discarded & L & - & - & $\begin{array}{l}\text { uses a curated } \\
\text { full-length human } \\
\text { ERV database }\end{array}$ & 157 \\
\hline $\begin{array}{c}\text { Random } \\
\text { assignment of } \\
\text { multi-mappers }\end{array}$ & $\begin{array}{l}\text { Reference } \\
\text { genome }\end{array}$ & $\begin{array}{l}\text { randomly } \\
\text { assigned }\end{array}$ & L & - & - & $\begin{array}{l}\text { locus-specific } \\
\text { transcription not } \\
\text { reliable on } \\
\text { youngest TEs }\end{array}$ & 134 \\
\hline TEcandidates & $\begin{array}{l}\text { Reference } \\
\text { genome }\end{array}$ & $\begin{array}{l}\text { remapped on } \\
\text { partially } \\
\text { masked } \\
\text { reference } \\
\text { genome } \\
\end{array}$ & L & - & - & - & 146 \\
\hline SQuIRE & $\begin{array}{l}\text { Reference } \\
\text { genome }\end{array}$ & EM algorithm & L & - & $+/-$ & $\begin{array}{c}\text { polymorphic } \\
\text { insertion can be } \\
\text { added as extra } \\
\text { chromosome if } \\
\text { internal sequence } \\
\text { known }\end{array}$ & 145 \\
\hline $\begin{array}{l}\text { Manual } \\
\text { curation }\end{array}$ & $\begin{array}{l}\text { Reference } \\
\text { genome }\end{array}$ & discarded & L & + & - & $\begin{array}{l}\text { difficult to } \\
\text { generalize }\end{array}$ & 99 \\
\hline Telescope & $\begin{array}{l}\text { Reference } \\
\text { genome }\end{array}$ & EM algorithm & L & + & - & - & 149 \\
\hline L1EM & $\begin{array}{c}\text { Reference } \\
\text { genome and } \\
\text { model } \\
\text { transcriptome }\end{array}$ & EM algorithm & L & + & - & $\begin{array}{l}\text { proof-of-principle } \\
\text { on human L1s, } \\
\text { could be } \\
\text { generalized } \\
\end{array}$ & 65 \\
\hline Multi-omics \#1 & $\begin{array}{l}\text { Reference } \\
\text { genome }\end{array}$ & NA & L & + & + & $\begin{array}{c}\text { combines } \\
\text { targeted DNA } \\
\text { sequencing, } \\
\text { RNA-seq and } \\
\text { ChIP-seq }\end{array}$ & 154 \\
\hline Multi-omics \#2 & $\begin{array}{l}\text { Reference } \\
\text { genome }\end{array}$ & NA & L & + & + & $\begin{array}{l}\text { combines whole- } \\
\text { genome } \\
\text { sequencing and } \\
\text { RNA-seq } \\
\end{array}$ & 33 \\
\hline
\end{tabular}

ChIP-seq, chromatin immunoprecipitation followed by sequencing; EM, expectation maximization; F, family-specific; L, locus-specific, RNA-seq, RNA sequencing; TE, transposable element; NA, not applicable. 
a ERV and LTR retrotransposons

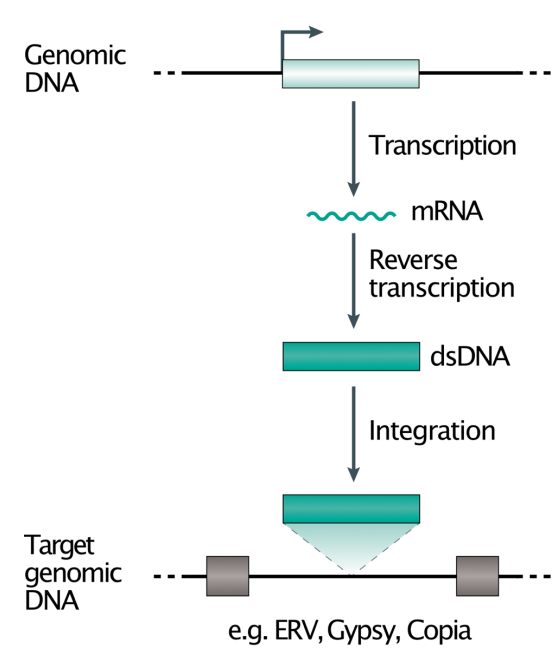

b Non-LTRretrotransposons

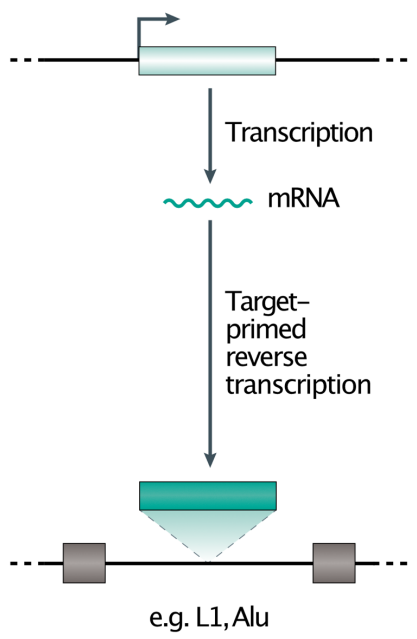

e.g. L1,Alu
C DNA transposons

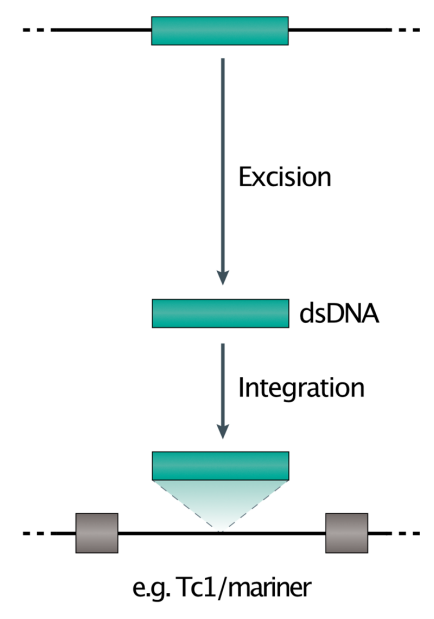

Class Il: cut and paste

Class I: copy and paste

Fig. 1. Transposable element classes and their intermediates. Since the end of the 1980s, transposable element (TE) classification has evolved continuously ${ }^{207-209}$. TEs are generally divided into two main classes depending on their mobilization mechanism and molecular intermediates. Class I comprises retroelements that replicate through an RNA intermediate and a reverse transcription step, the so-called copy-and-paste transposons, and comprises two main families: endogenous retroviruses (ERVs) and long terminal repeat (LTR) retrotransposons, such as Gypsy and Copia elements (a) and non-LTR retrotransposons, such as long and short interspersed elements (LINE and SINE, respectively) (b). The reverse transcription of ERVs and LTR retrotransposons occurs in cytoplasmic viral-like particles and leads to the formation of extrachromosomal double-stranded DNA (dsDNA), which is imported into the nucleus before integrating into a new locus. Non-LTR retrotransposons initiate reverse transcription directly at the target locus after cleaving genomic DNA, a process known as target-primed reverse transcription (TPRT). DNA transposons, the so-called cut-and-paste transposons, form class II (c). Their mobilization involves the excision of the transposon DNA from its original locus and its re-integration into another locus. Each class of TE comprises autonomous and non-autonomous elements. Autonomous elements encode the enzymes necessary for their own mobilization, whereas non-autonomous elements hijack the machinery encoded by autonomous elements. Other less-represented or studied families have been described, such as Helitrons, Crypton and Maverick (not shown). Molecular details of mobilization mechanisms have been reviewed elsewhere ${ }^{209,210}$. 
a Major postintegration alterations

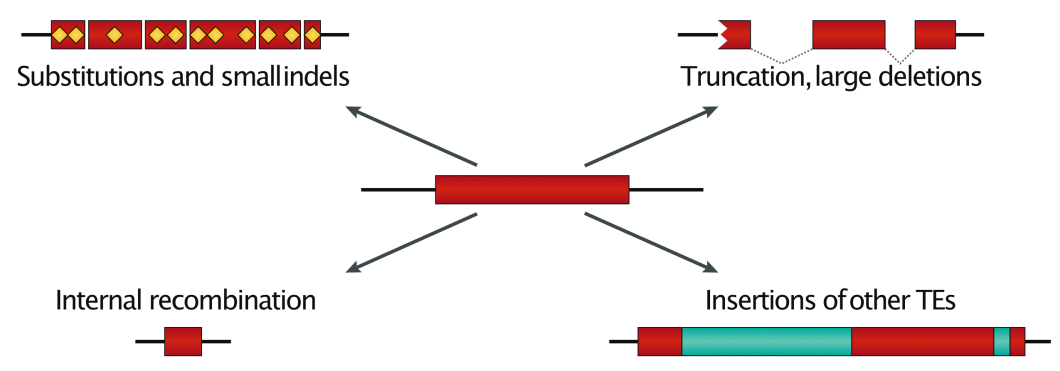

b Build TE consensus sequences

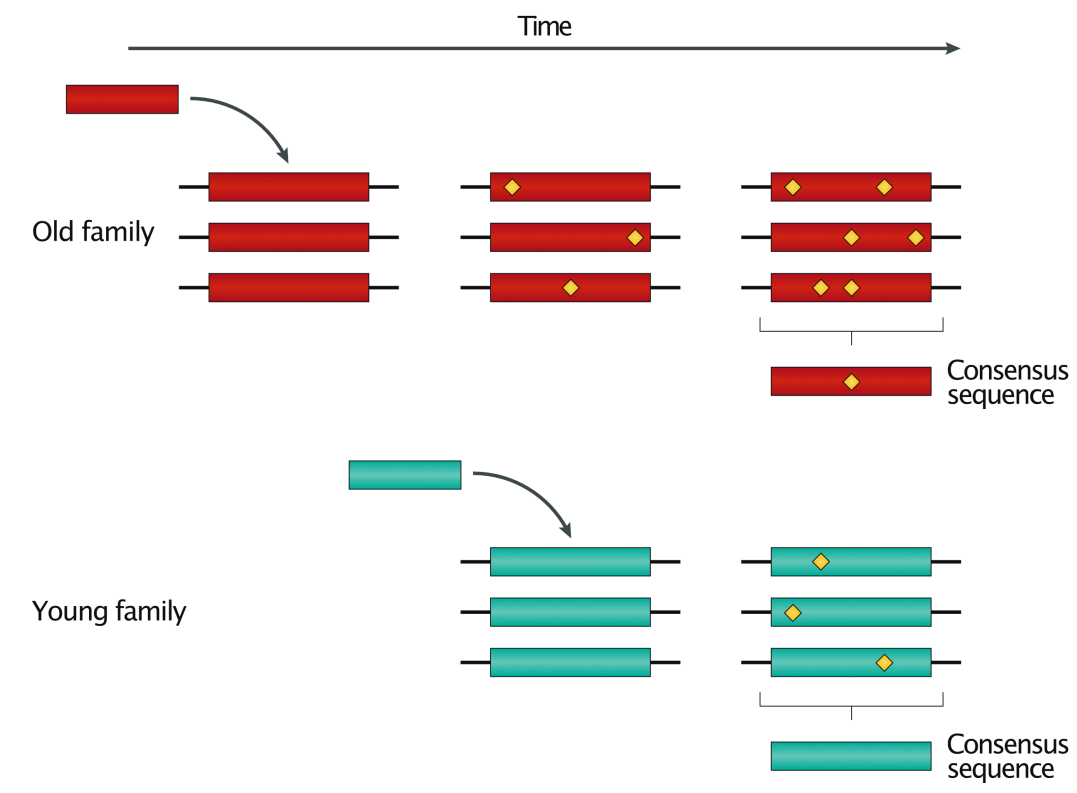

c Age distribution of TEs in a given genome

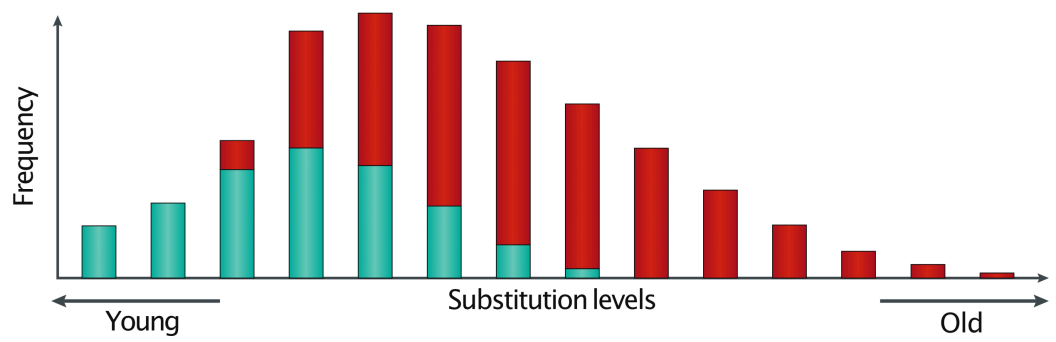

Fig. 2. Post-integration alterations and transposable element consensus sequences. Active progenitor transposable elements (TEs) can lead to multiple new insertions, all identical or nearly identical to each other. a. In the absence of positive selection, TE copies progressively diverge after integration through a variety of processes, such as substitutions, small insertions and deletions (diamonds, indels), truncations or large deletions, recombination between terminal repeats (for example, between long terminal repeat (LTR), leading to soloLTR), or insertion of other TEs (green). The extent of these alterations depends on the time since integration. b. Consensus sequences can be built for each TE family by aligning the individual copies (red and green) that contain substitutions (diamonds). These consensus 
sequences are centralized in databases such as Repbase ${ }^{135}$, Dfam ${ }^{211}$ or RepetDB ${ }^{212}$. Although these model sequences do not generally exist in real genomes, they can be considered a rough reconstruction of the ancestral progenitor element. c. Consensus sequences are essential for annotating genomes and can be used to calculate the genetic distance approximated by the level of substitutions (most often the CpG-adjusted Kimura substitution levels) - between TE copies of the same family, and to estimate insertion time (graph). 

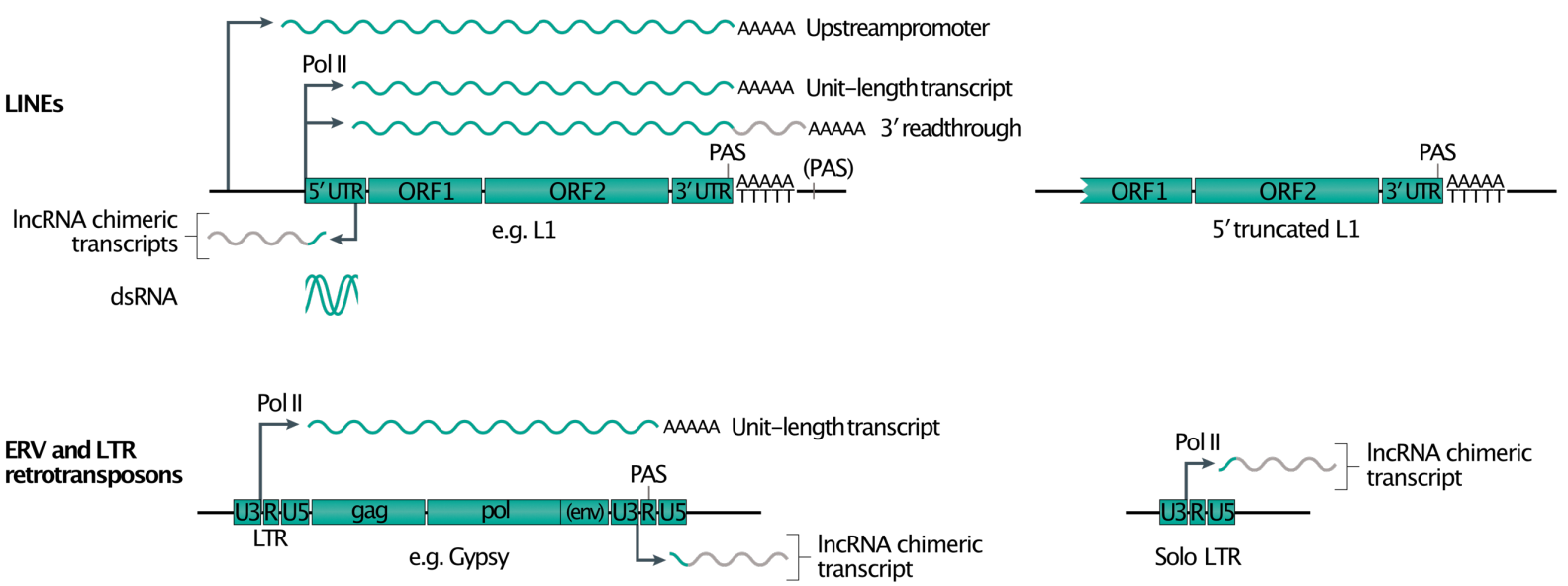

SINEs
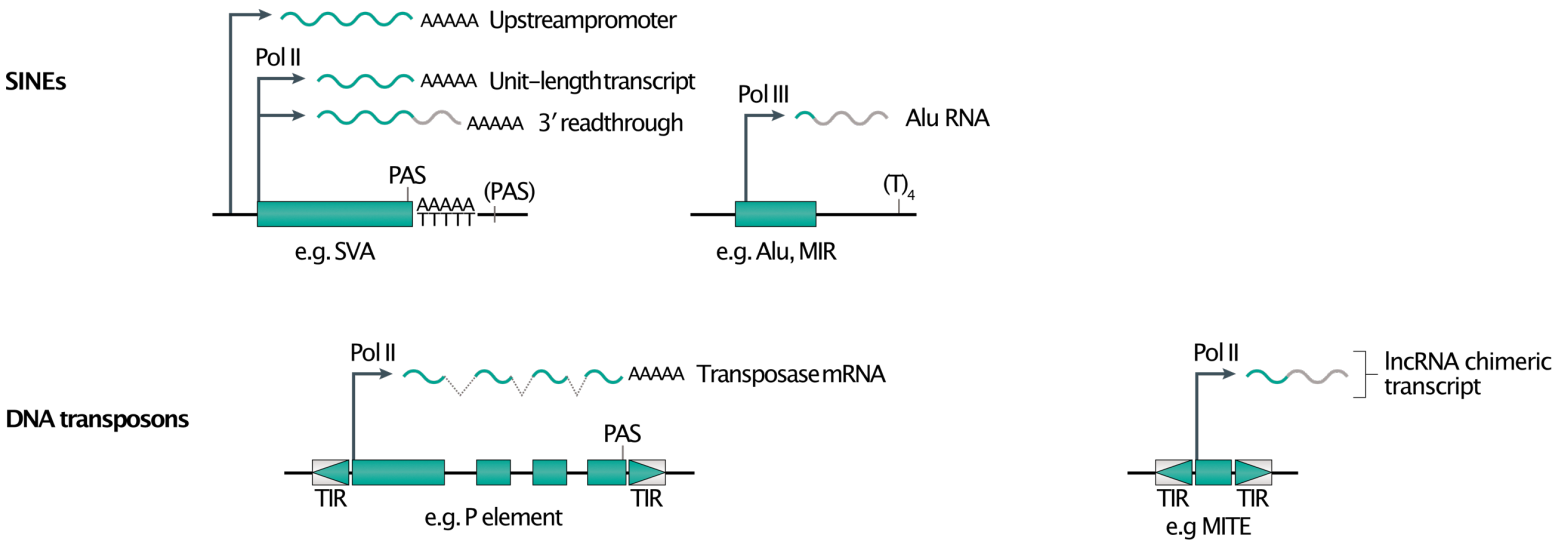

b Interactions between the transcription units of genes and TEs

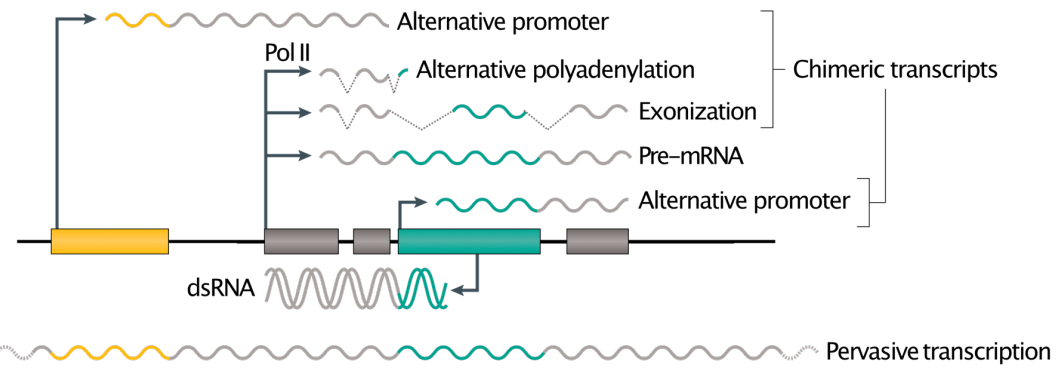

Fig. 3. Origins of TE-derived transcripts. a. The left panel shows the different transcriptional units for the main TE families and the different structures of their transcripts. Autonomous transcription of TEs can be promoted by RNA polymerase II (Pol II) or Pol III and terminates at the polyadenylation signal (PAS) presents at their 3' extremity. Antisense or 3' promoters can promote long non-coding RNA (IncRNA) or chimeric transcripts synthesis while the convergent transcription (antisense and sense transcription) can induce the formation of double-stranded RNA (dsRNA). The right panel illustrates other representative TE forms for each family. b. The lower panel illustrates possible chimeric transcripts between TE (orange 
and green) and genic transcription units (grey). Dotted lines represent spliced intronic sequences. LTR, long terminal repeat, ERV, endogenous retrovirus, PAS, polyadenylation signals, IncRNA long non-coding RNA, dsRNA, double-stranded RNA, ORF, Open Reading Frame, TIR, terminal inverted repeat. 

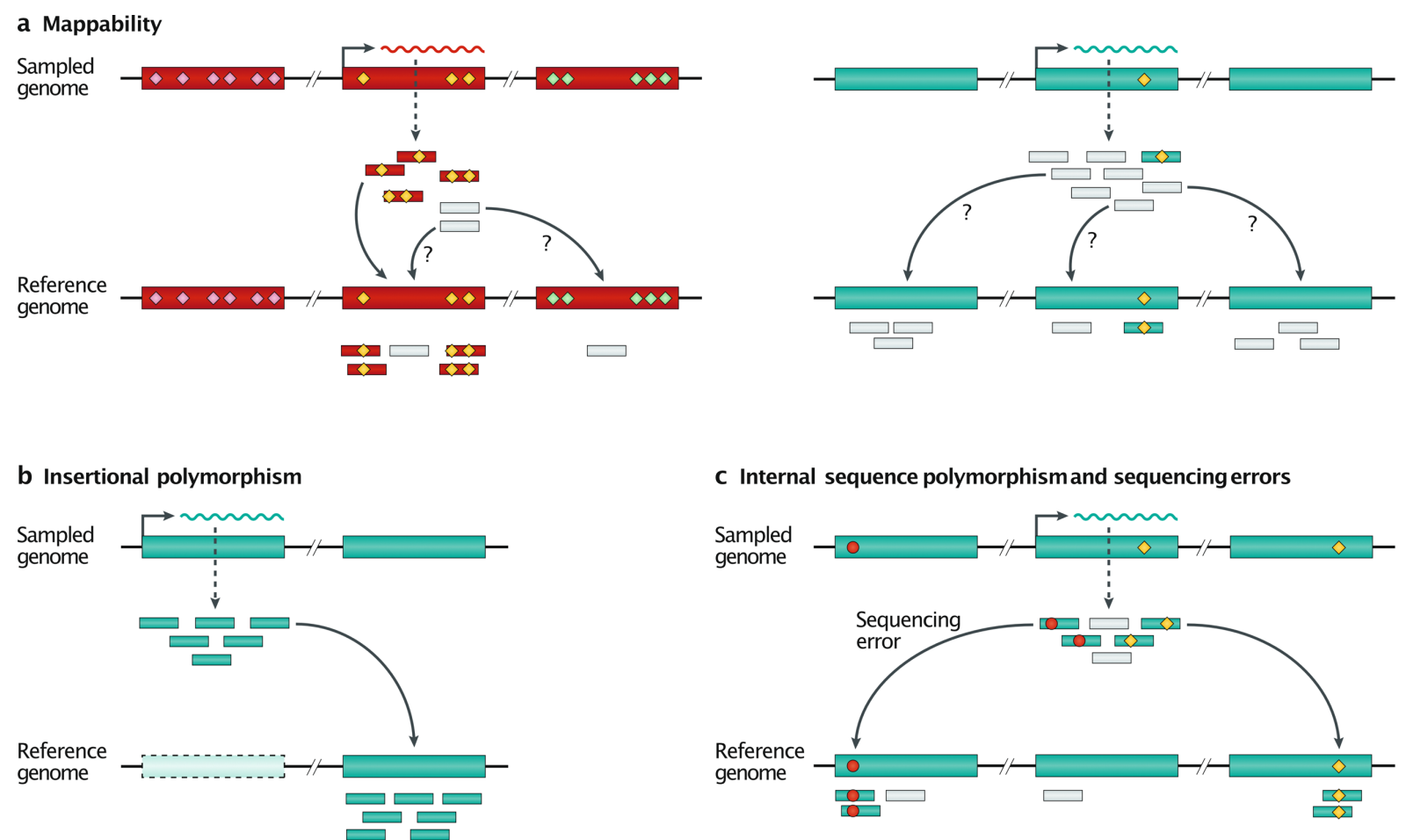

Fig. 4. Origins of ambiguous mapping. a | Mappability. Old insertions (red rectangles) have accumulated discriminative SNPs (diamonds) as compared to younger elements (green rectangles). Consequently, young TE-derived reads (green bars) tend to map at multiple positions in the genome (light grey bars, multi-mappers), and their true locus of origin cannot be defined. In contrast, more uni-mappers (filled bars) can be unambiguously mapped at older elements, facilitating the quantification of their expression. Multi-mappers were randomly assigned. b | Insertional polymorphisms. A TE present in the genome of the studied sample (light green, top) but absent from the reference genome (dashed rectangle, bottom) can be expressed. However, reads being mapped to the reference genome, they will be incorrectly assigned to a reference TE copy despite being uni-mappers. c | Internal sequence polymorphisms and sequencing errors. In the reference genome pictured (bottom), each individual copy has a discriminative SNP (circle and diamond). However, in the studied genome (top), the right locus also possesses the diamond SNP. In addition, sequencing errors lead to the incorporation of the circle SNP in a fraction of the reads emanating from the rightmost locus. This situation results in mis-mapping of the reads to the left and right loci, instead of the expressed middle locus. Multi-mappers were randomly assigned. 


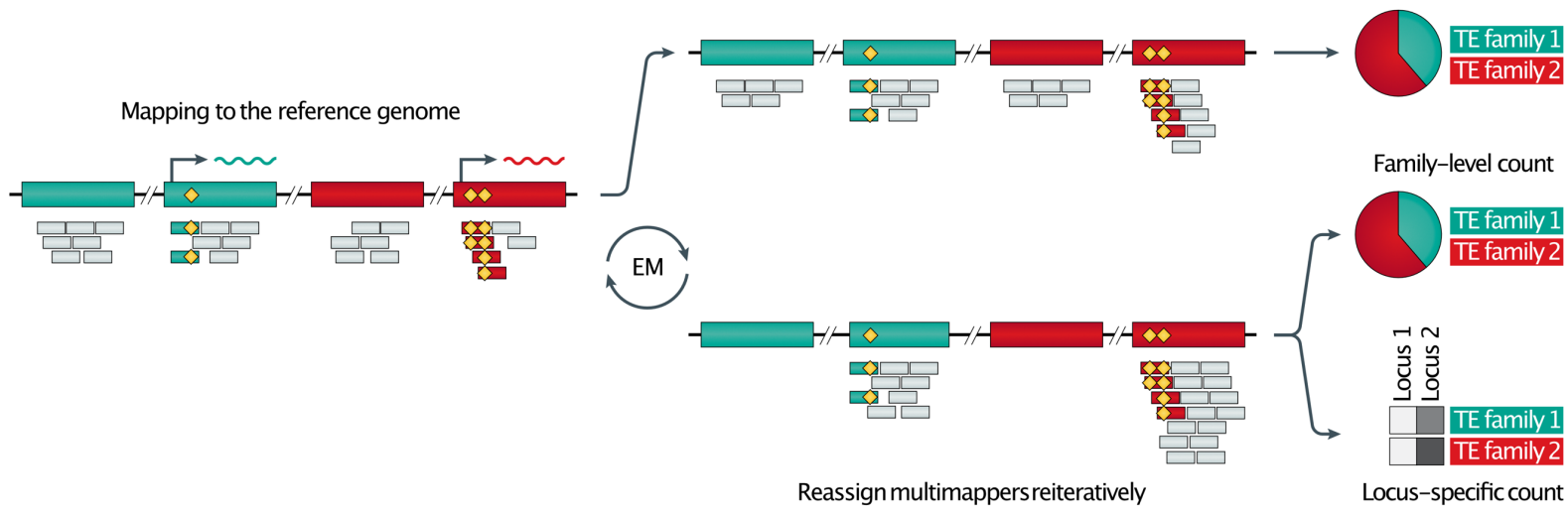

b Pseudomapping on a model transcriptome

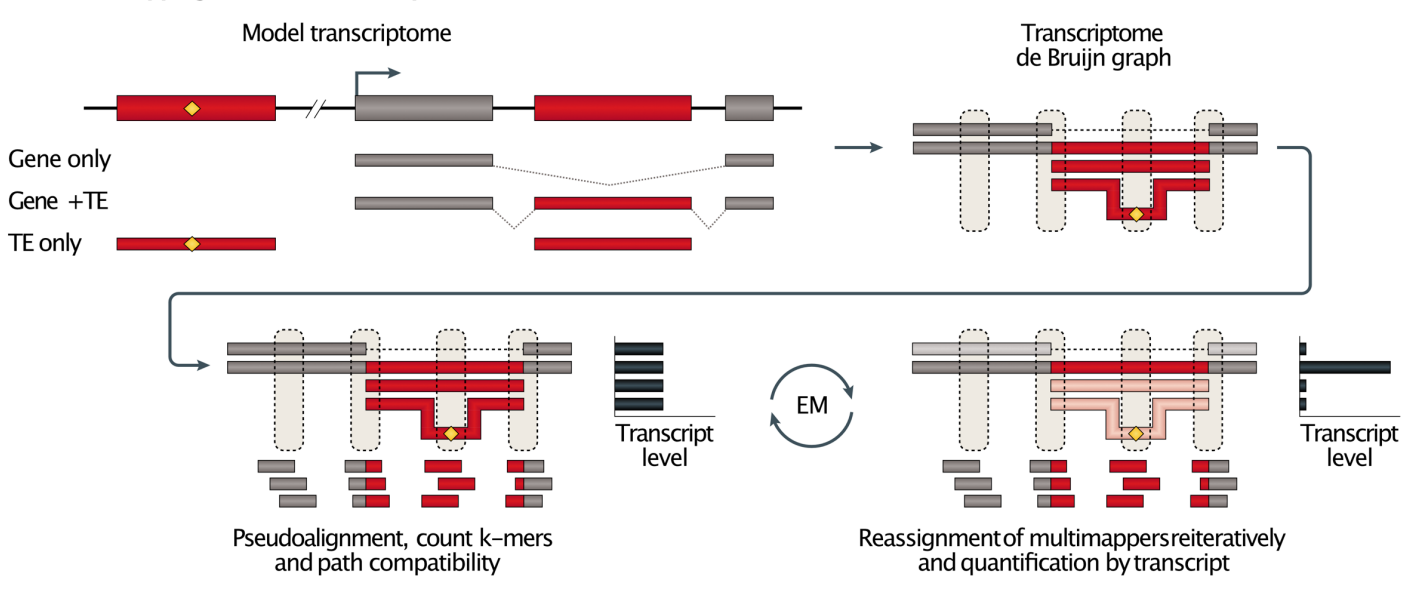

C Hybrid mapping on a reference genome and model transcriptome
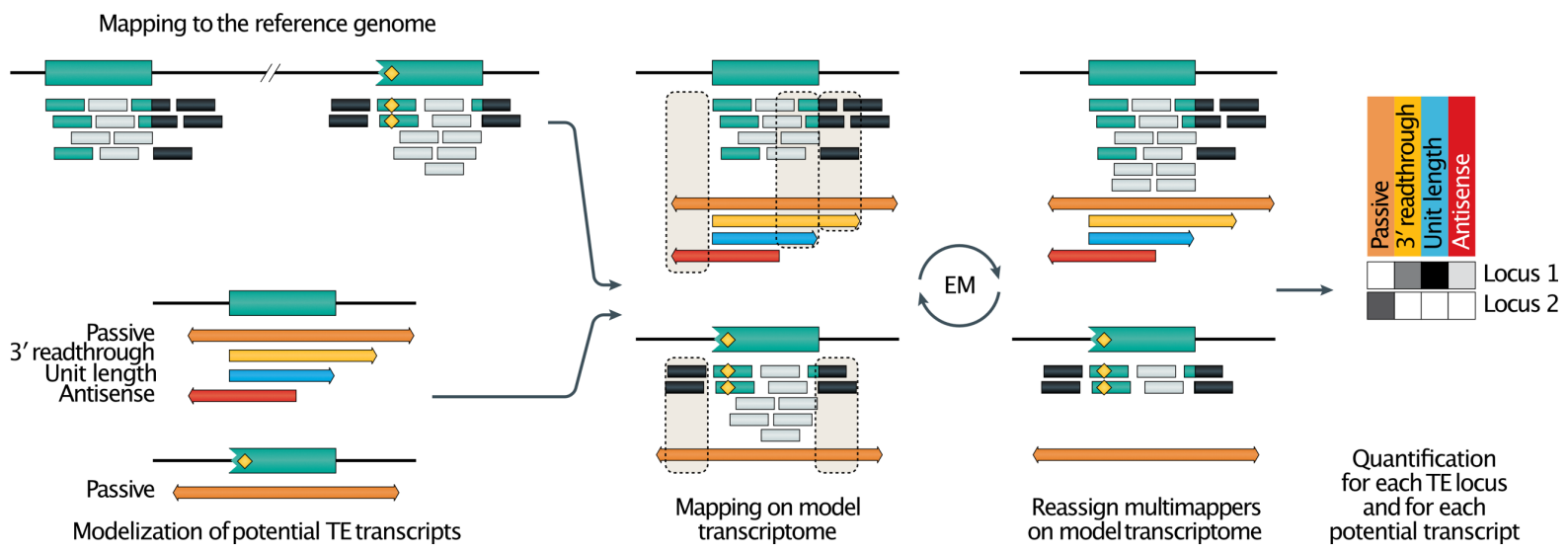

Fig. 5. Recent strategies to measure TE expression from RNA-seq data. (a) Mapping on a reference genome. Strategies differ by the way multi-mapper (light grey bars) are handled: (top) random assignment of multi-mappers among best matching TE copies (represented by green and red rectangles) and aggregation of read counts by TE family (e.g. ${ }^{134}$ ); (bottom) application of the expectation maximisation (EM) algorithm to statistically redistribute multimappers reiteratively. This can be followed also by family-level aggregation of read counts (e.g. TEtranscripts ${ }^{148}$ ) or can render locus-specific read count (e.g. SQulRE ${ }^{145}$ ). 
Pseudomapping on a model transcriptome. Potential transcripts originating from each TE locus are included in the model transcriptome. In the simplified model shown here, a family is represented by two TE loci (red rectangles): first an intergenic copy with a discriminative SNP (orange diamond, left), and second, an intronic insertion (right) embedded in a gene (grey). The left TE has only a single potential unit-length transcript ('TE only'), while the right locus can be expressed as 3 alternative transcripts (TE only, 'gene + TE', or 'gene only'). From this model transcriptome, an index is built by creating the transcriptome de Bruijn Graph (T-DBG) where each node (dotted ovals) are k-mers (short sequences with a length of k nucleotides) informative of the specific isoform transcribed. Pseudo-alignments as performed by Kallisto or Salmon extract k-mers from RNA-seq reads, test their compatibility for each node and find the "path covering" in the T-DBG (here only 'gene + TE' is covered). Then, the EM algorithm is used to reassign ambiguous k-mers reiteratively and to quantify reads at a family level according to the sub-localisation (intronic, exonic and intergenic) (e.g. REdiscoverTE ${ }^{153}$ ). (c) Hybrid mapping on a reference genome and model transcriptome. A model transcriptome representing the different potential transcript isoforms at each TE locus is built for all full-length element (passive in orange, 3'readthrough in yellow, autonomous in blue and antisense transcription in red). Only pervasive transcription is included for truncated elements. A diagnostic SNP is shown in the full-length element (orange diamond). Reads are first aligned to the reference genome and then those affected to TE loci, including multi-mappers, are mapped on the model transcriptome, reassigned with the EM algorithm. Quantification is obtained for each TE locus and for each associated transcript isoform (e.g. L1EM ${ }^{65}$ ). 


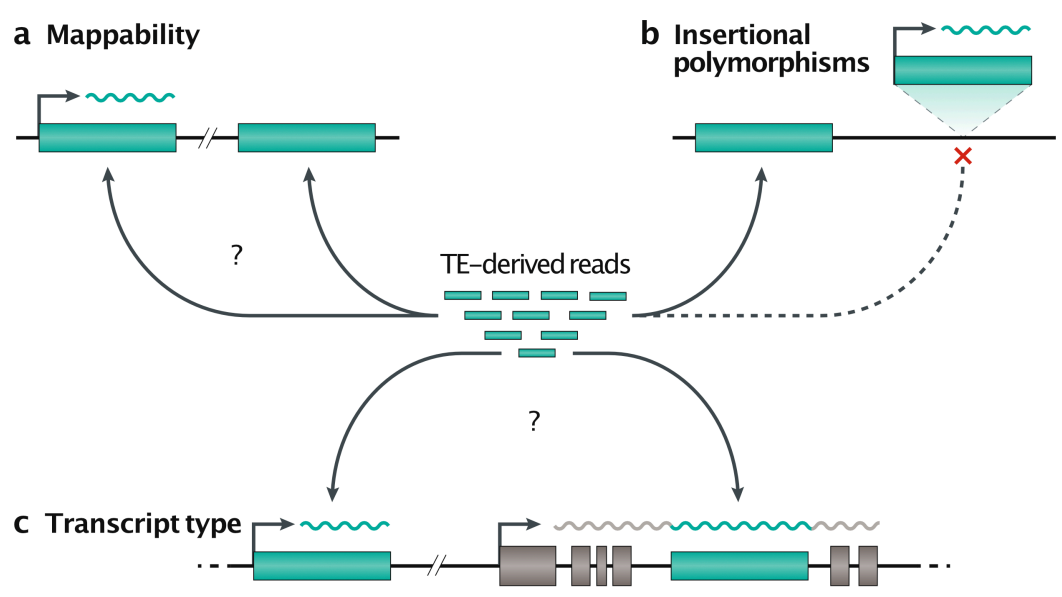

Fig. 6. Challenges associated with the study of TE transcription. TE transcriptional studies are facing three major difficulties: (a) mappability, (b) polymorphisms, and (c) transcript type. (a) Recently inserted TEs show low sequence divergence among individual copies or within close families. Consequently, TE-derived reads can align to multiple genomic positions with identical scores. $\mathbf{b}$. The ongoing mobilization of some TE families leads to a high diversity of integration sites and creates polymorphic TE insertions in populations, not included in the reference genome. Reads derived from such polymorphic TE insertions are incorrectly mapped to the closest related loci represented in the reference genome, overestimating the expression of the latter. Internal sequence polymorphisms at a given TE locus and variable between individuals are another source of ambiguous mapping (not shown). (c) The autonomous transcription of TE unit-length transcripts (left) can be easily confounded with TEchimeric transcripts or with the expression of the gene into which a given TE is inserted (right), affecting experimental interpretation. 


\section{Box 1. Technical considerations}

The architecture and origin of TE-containing transcripts are diverse and reflect a wide range of biological processes. These are sometimes difficult to distinguish and, therefore, the levels of TE expression, or their variation, can be misinterpreted.

For both RNA-seq and hybridization-based experiments, strand-specific assays are essential to reliably infer the structure of the transcript and its origin. Similarly, the nature of the starting RNA material can strongly influence the conclusion that can be drawn. The use of total RNA, a common practice in reverse transcription-quantitative PCR (RT-qPCR) experiments, is uninformative as it will indiscriminately quantify unit-length transcripts, potential chimeric transcripts, intronic and exonic co-transcripts, and pervasive transcription. By contrast, isolation of polyA-positive RNA from whole cells or cytoplasmic RNA can enrich mature mRNAs and reduce the contribution of intronic TEs or pervasive transcription to the observed signal ${ }^{100,159}$. Alternatively, the use of rRNA-depleted RNA can reveal nonpolyadenylated long non-coding RNAs (IncRNAs) with important regulatory roles that cannot be detected if only polyA-positive RNAs are sequenced ${ }^{213,214}$. Thus, the choice of the starting material should be guided directly by the biological questions asked.

Another underappreciated pitfall when measuring TE RNA levels is genomic DNA contamination. A small amount of contaminating DNA, which would not greatly affect the measurement of gene expression, could significantly influence TE expression results owing to the high TE copy number. In addition, these contaminations are generally not reproducible and can vary considerably from sample to sample. This can easily be verified in RT-qPCR experiments by including RT-minus control samples. For RNA-seq, checking the consistency of intron-exon or intergenic-intragenic signal ratios can help to identify poor-quality samples $^{159}$. Biases resulting from DNA contamination are not limited to total RNA or rRNAdepleted RNA. Indeed, the oligo(dT) used to pull down polyA-positive RNA can potentially pull down DNA fragments with long poly $(\mathrm{dA})$ tracts as found at the 3' end of many non-LTR retroelements, such as L1, Alu or SVA elements. To limit these problems, we recommend to perform two successive rounds of RNA purification (by acid phenol-guanidinium thiocyanate or silica-based column), followed by DNase digestion. 


\section{Box 2. Mappability, alignability and uniqueness}

Mappability can be estimated through two distinct metrics: alignability and uniqueness. Alignability, is defined by the frequency of a sequence found at a specific location to align somewhere else in the genome ${ }^{215}$. Generally, mismatches are tolerated up to a certain extent (for example, two mismatches) to account for sequencing errors or SNPs. Briefly, alignability can be estimated throughout a reference genome by (i), generating simulated sequencing reads with a defined length, (ii), mapping back these virtual reads onto the reference genome, allowing some mismatches, and (iii), calculating the number of positions to which these reads map. For example, if a read generated from a locus has five distinct matches in the genome, its alignability will be $1 / 5=0.2$. Uniqueness is similar, but no mismatch is tolerated, and the score is set as 0 for more than four alternative locations, such as in the example above.

Low-complexity regions and repeated sequences such as TEs exhibit low mappability. Obviously, read length strongly influences the mappable fraction of a genome. In addition, the mappability of a TE family/insertion is correlated to its age ${ }^{133}$. Young elements display a lower mappability score, which can considerably bias TE sequencing studies. Thus, estimating their mappability can be useful to assess which TE family or locus can be confidently quantified at the locus-specific level rather than at the aggregated family level ${ }^{133}$. Similarly, low mappability regions are also prone to artefactual mapping even when only considering uni-mappers due to genetic variation or sequencing error ${ }^{216}$. Mappability scores calculated from different read lengths across human and mouse genomes, including their TEs, can be obtained from UCSC genome browser website. 


\section{Glossary}

Polymorphic: A term often used for TE insertional polymorphisms, whereby a TE insertion can be present or absent at a given locus or allele in a subset of individuals from the same species.

Autonomous TE unit transcription: TE transcription driven by its own internal promoter.

TE unit-length transcripts: Full-length TE transcripts that can serve as template for reverse transcription to produce a new intact copy.

TE-chimeric transcripts: Transcripts containing both TE and non-TE (typically a gene) sequences.

Pervasive transcription: Transcription of regions well beyond the boundaries of known genes.

Co-transcription: Intronic TE expression through the expression of its surrounding gene without the implication of the promoter activity of the TE. Synonymous to readthrough transcription.

Multi-mappers: Sequencing reads that map ambiguously at multiple locations in the reference genome.

Uni-mappers: Sequencing reads that can map non-ambiguously to a single location in the reference genome.

LTR-retrotransposons: A class of retrotransposons that contains two long repeated sequences in direct orientation at both ends.

Positive selection: A type of natural selection that promotes the spread of a beneficial trait or genetic variant within a given population.

k-mers: Short sequences of a length of $k$ bases.

\section{ToC blurb}

Computational tools to analyse RNA-sequencing data often disregard or even misinterpret reads derived from transposable elements (TEs). This Review highlights the main challenges associated with the detection of TE expression, including mappability, sequence polymorphisms and transcript diversity, and discusses the experimental and computational strategies to overcome them. 